\title{
Effect of Brittle off-fault Damage on Earthquake Rupture Dynamics
}

\author{
Marion Y. Thomas ${ }^{1,2}$, Harsha S. Bhat ${ }^{1}$, Yann Klinger ${ }^{1}$ \\ ${ }^{1}$ Institut de Physique du Globe de Paris, Paris, France. \\ ${ }^{2}$ Department of Earth Sciences, University of Oxford, Oxford, UK.
}

\section{Key Points:}

- Dynamic damage is different in the compressional and tensional quadrant and is sensitive to material contrast.

- Off-fault medium history (asymmetric damage pattern) influences earthquake rupture processes.

- Dynamic damage induces a reduction in elastic moduli and slip rate oscillations.

- Dynamic damage produces high frequency content in the radiated ground motion. 


\begin{abstract}
In the shallow brittle crust, following earthquake ruptures, geophysical observations show a dramatic drop of seismic wave speeds in the shallow off-fault medium. Seismic ruptures generate, or reactivate, damage around faults that alter the constitutive response of the surrounding medium, which in turn modifies the earthquake itself, the seismic radiation and the near-fault ground motion. This numerical study aims to assess the interplay between earthquake ruptures and dynamically evolving off-fault medium and to underline the damage-related features pertinent to interpret geophysical observations. We present a micro-mechanics based constitutive model that account for dynamic evolution of elastic moduli at high-strain rates. We consider 2-D inplane models, with a 1-D right lateral fault featuring slip-weakening friction law. We demonstrate that the response of the damaged elastic solid is different in the compressional and tensional quadrant. We observe that dynamic damage induces a reduction in elastic moduli and produces slip rate oscillations which result in high frequency content in the radiated ground motion, consistent with strong motion records. We underline the importance of incorporating off-fault medium history in earthquake rupture processes. We find that dynamic damage generation is sensitive to material contrast and that it introduces an additional asymmetry beyond that of a bimaterial fault, in agreement with experimental studies.
\end{abstract}

\title{
1 Introduction
}

Deformation of natural fault zones in the brittle crust ( $\sim 0$ to $40 \mathrm{~km}$ depth) is conventionally perceived of that of two planes, sliding one against the other, loaded by constant slip at greater depth, and whose behavior is controlled by the frictional properties of the interface [Scholz, 1998]. Depending on these properties, when the frictional resistance is overcome, the accumulated stress is released by stable sliding or by unstable dynamic events. As a consequence, in recent years, numerous studies have been carried out to determine these properties for various settings [e.g., Byerlee, 1978; Scholz, 1998; King and Marone, 2012; den Hartog et al., 2012]. However, if the behavior of fault zones is intrinsically linked to the properties of the fault interface, it also depends on those of the surrounding medium [e.g., Andrews, 2005]. Fault not only consist of a fine-grained narrow fault core where slip occurs, but it is also surrounded by pervasively fractured rocks, within a complex 3-D geometry.

Sibson [1977] contribution is largely recognized as the first attempt to give a coherent description of an active fault zone. Scholz [2002] further proposed a slightly different fault rock classification, which is widely used nowadays. Following the enticing review by Biegel and Sammis [2004], one can use the Punchbowl fault zone as a representative model of "mature" strike-slip faults that have recorded large displacement. Fault zone may be then idealized as an intricate structure consisting of a fault core, surrounded by a damage zone. Based on the revised description of the Punchbowl fault structure by Chester et al. [1993], the fault core comprises an inner layer of ultracataclasite bounded by an outer layer of foliated cataclasite. The extremely fine grained core material is then surrounded by a damage zone that includes layers of gouge and breccia bordered by fractured rocks. The last two layers are included in the damage zone because they lacked extensive shearing [Chester et al., 1993; Biegel and Sammis, 2004]. Evidently, there are significant variations from one fault zone to another, but they share in common a highly fine grained fault core (often extremely narrow band), where most of the displacement have occurred, surrounded by a damaged wall rock. However, the gouge and breccia layer is missing along various faults [Biegel and Sammis, 2004].

Systematic micro- and macrostructural field studies have recently been performed on damage zones [Shipton and Cowie, 2001; Faulkner et al., 2006; Dor et al., 2006; Mitchell and Faulkner, 2009; Faulkner et al., 2011; Savage and Brodsky, 2011], as a key component to understand the energy balance of earthquakes [e.g., Rice, 2002; Kanamori, 2006]. The width of the damage zone is determined by measuring the decay in crack intensity away from the fault core, until it falls to the background level of the host rock [e.g., Chester and Logan, 1986; Biegel and Sammis, 2004; Faulkner et al., 2011]. The fractured rocks usually have a 
spatial scale of the order of metres to kilometres, however this is difficult to define and particularly time-consuming, hence there are few field surveys focused on the structure of the damage zone. Among them, several studies in low-porosity rocks (crystalline, sedimentary rocks) have highlighted an exponential decay of cracks density away from the fault [Vermilye and Scholz, 1998; Wilson et al., 2003; Mitchell and Faulkner, 2009; Faulkner et al., 2006, 2011], or alternatively, that the decay could be fit using a power law [Savage and Brodsky, 2011]. As underlined by Faulkner et al. [2011], the trends is less clear for faults developed in higher-porosity rocks [Shipton and Cowie, 2001]. Possible mechanisms responsible for the development of off-fault damage could include the fault geometry, linking of structures, the quasi-static stress field, the process zone associated with fault growth and the coseismic fracture damage [Vermilye and Scholz, 1998; Rice et al., 2005; Childs et al., 2009; Faulkner et al., 2011; Vallage et al., 2015]. In their study of the Bolfín fault in northern Chile, Faulkner et al. [2011] have discussed several processes that could explain the development of the observed scaling inside the fault zone and concluded that the spatial extent of damage might be better explained by the damage zone growth with increasing displacement, due to geometric irregularities, or by coseismic damage.

Fault zone structure is of key importance in the mechanics of faulting. In fact, several studies have underlined the importance of the fault zone fabric in controlling the slip behavior of fault zones [Collettini et al., 2009; Niemeijer et al., 2010; Thomas et al., 2014a; Audet and Burgmann, 2014; Klinger et al., 2016]. For example, Audet and Burgmann [2014] have recently highlighted a direct relation between the properties of the overlying forearc crust in subduction zones and the average recurrence time of slow earthquakes. Along the Longitudinal Valley Fault in Taiwan, a structural analysis and a kinematic study have demonstrated that the damaged forearc formation favors aseismic creep whereas the locked segments of the fault are in contact with intact rocks, or the protolith [Thomas et al., 2014b,a]. Fault zone structure is equally important during seismic slip. The complexity of the fault zone system impacts the rheological properties of the fault core and the surrounding medium, both of which influence the seismogenic behavior of the fault. The changes in elastic stiffness of the bulk control the amount of elastic strain energy that can be stored during tectonic loading and released during earthquakes and can induce a stress rotations (due to contrasting elastic moduli with the host rock), allowing faults to slip under less optimal far-field stress conditions [Faulkner et al., 2006]. During seismic faulting, the stress concentration at the tip of the rupture generate, or reactivate, damage (fractures) around faults that modifies the microstructure and the constitutive response of the surrounding medium [e.g., Rice et al., 2005]. Seismic ruptures can trigger a significant coseismic drops in velocity (reduction in elastic stiffness of up to 40\%), on spatial scales of hundreds of metres normal to the fault and few kilometres along depth, followed by time-dependent recovery over couple of years [Hiramatsu et al., 2005; Li et al., 2006; Brenguier et al., 2008; Cochran et al., 2009; Froment et al., 2014]. In turn, the coseismic change in elastic moduli influences the rupture itself, which has a direct effect on the size of the earthquake, the radiated waves field and near-fault ground motion [Walsh, 1965a,b; Faulkner et al., 2006; Bhat et al., 2012]. Therefore the evolution of damage during earthquake is critical to understand the nucleation, propagation and arrest of earthquakes. This numerical study in particular aims to explore intricate feedbacks between the spontaneous off-fault damage generation and the dynamic rupture propagation.

Numerous studies in the last couple of decades have explored the effect of off-fault plasticity on seismic rupture, using either analytical approaches [Rice et al., 2005; Ngo et al., 2012] or numerical simulations (see following references). Some models have explored the effect of damage on the properties of the dynamic rupture (mode, speed and directivity) and final slip by prescribing a low-velocity zone around the fault [e.g., Kaneko and Fialko, 2011; Cappa et al., 2014; Huang et al., 2014]. Another set of models uses a Mohr-Coulomb [e.g. Andrews and Harris, 2005; Ben-Zion and Shi, 2005; Hok et al., 2010; Gabriel et al., 2013] or Drucker-Prager [e.g. Templeton and Rice, 2008; Ma, 2008; Dunham et al., 2011] yield criterion to investigate dynamic rupture propagation with spontaneous dynamic formation of off-fault damage. In their study, Yamashita [2000] and [Dalguer et al., 2003] model the gen- 
eration of off-fault damage as the formation of tensile cracks, using respectively a stress- and fracture-energy-based criterion. If these types of study provide a good insight on the effect of damage structure on seismic rupture, they do not account for dynamic changes of elastic moduli in the medium and therefore do not completely model the feedbacks between the off-fault damage and the seismic rupture. Determining the constitutive behavior of the surrounding medium requires developing models based on the mechanics of cracks, and how they response to the applied loading. It requires to use an energy-based approach to develop the new constitutive law [e.g., Lyakhovsky et al., 1997a; Finzi et al., 2009; Suzuki, 2012; Xu et al., 2014; Lyakhovsky and Ben-Zion, 2014]. In particular, these models need to include a physical crack growth law to model the evolution of damage. Ideally, this law should incorporate the loading rate crack-tip velocities dependency of fracture toughness [Chen et al., 2009; Dai et al., 2010, 2011; Wang et al., 2010, 2011; Zhang and Zhao, 2013], which is particularly important to model earthquake-related processes. The latter constitutes the essential difference between the model presented in this paper and the models aforementionned.

The development of the constitutive model is presented section 2, followed by a description of the numerical method and the parameters we used for this paper. In the third section we consider three different scenario to explore the interplay between earthquake rupture and offfault damage, how it affects both the hosting medium and the rupture propagation, and what are the damage-related features that can be pertinent to interpret geophysical observations. Our findings are summarized in section 5.

\section{Constitutive Model}

This numerical study aims to explore the effect of spontaneous off-fault damage generation on dynamic rupture propagation. This section provides the description of the constitutive model used to account for the dynamic evolution of elastic properties in the surrounding medium, related to dynamic off-fault damage. The different parameters and constants used for the constitutive model are summarized in Table 1.

\begin{tabular}{lcc}
\hline Parameter & Symbol & Equation \\
\hline angle to $\sigma_{1}$ for microcracks & $\Phi$ & eq 12 \\
projection of $a$ to $\sigma_{1}$ & $\alpha$ & $\cos \Phi$ \\
Damage variable & $D$ & eq 13 \\
Initial Damage variable & $D_{0}$ & eq 11 \\
stress intensity factor & $K_{I}$ & eq 21, 27 \\
dynamic stress intensity factor & $K_{I}^{d}$ & Bhat [2012], eq 43 \\
dynamic initiation toughness & $K_{I C}^{D}$ & Bhat [2012], eq 45 \\
dynamic fracture toughness & $K_{I C}^{d}$ & Bhat [2012], eq 46 \\
instantaneous wing-crack speed & $v \equiv \mathrm{d} l / \mathrm{d} t$ & Bhat [2012], eq 50 \\
stress & $\sigma_{i j}$ or $\boldsymbol{\sigma}$ & eq 32, 35, 41 \\
stress invariant & $\sigma, \tau$ & Bhat [2012] eq 11 \\
strain & $\epsilon_{i j}$ or $\epsilon$ & eq 6, 15 \\
strain invariant & $\epsilon, \gamma$ & eq 30 \\
\hline
\end{tabular}

Table 1. Parameters of the damage constitutive model

\subsection{Energy-based approach: general considerations}

The micromechanical method used in this study followed an energy-based approach to determine the constitutive strain-stress relationship of a damaged solid, as defined by Rice [1971], Hill and Rice [1973] and Rice [1975]. In other words, to account for fracture dam- 
age we create an energetically equivalent solid. This formalism, thermodynamically argued, was developed to model the inelastic behavior at macroscopic scale that arises from specific structural rearrangements at microscale, such as twinning in crystals, grain-boundary-sliding, phase transformation or microcrack development. This approach relates inelastic deformation of a given solid to a sequence of constrained equilibrium states, characterized by the values of strain $\epsilon$, temperature $T$ and internal variables $\xi$ (such as damaged state). Then, the relation between these properties and macroscopic stress are determined by fixing the internal variables at their current values for each imaginary equilibrium state, for which elastic constitutive law can therefore be applied. In practice, internal variables will have a time-dependent evolution, determined by the local conditions, but the kinetic aspect of it is taken care of separately (see section 2.7). Hence, the formalism of equilibrium thermodynamics can be adopted and using the properties of thermodynamic potentials, local structural rearrangement can be related to corresponding changes in the macroscopic stress and strain state [Rice, 1971].

Let $\boldsymbol{\epsilon}$ denotes the strain tensor for an arbitrary equilibrium state and let $\boldsymbol{\sigma}$ be the corresponding stress tensor such that $\boldsymbol{\sigma} d \boldsymbol{\epsilon}$ is the work per unit volume for any virtual deformation $d \epsilon$. The variable $\xi$ denotes the current "inelastic" state of the material. Following the thermodynamic principles, we can therefore write:

$$
\boldsymbol{\sigma}=\boldsymbol{\sigma}(\boldsymbol{\epsilon}, T, \xi)
$$

The basic assumption is that work potential

$$
W^{H}=W^{H}(\epsilon, T, \xi)
$$

exists at each $\xi$ within the associated $\epsilon$ domain. Then, for any strain variation $\delta \epsilon$ at fixed $\xi$, i.e., for which we obtain purely elastic deformation, we can write:

$$
\boldsymbol{\sigma} \delta \boldsymbol{\epsilon}=\delta W^{H}(\epsilon, T, \xi)
$$

and therefore, we can determine the stress tensor as followed:

$$
\boldsymbol{\sigma}=\frac{\partial W^{H}(\boldsymbol{\epsilon}, T, \xi)}{\partial \boldsymbol{\epsilon}}
$$

Within the elastic domain for any given $\xi, W^{H}$ actually corresponds to the Helmotz free energy [Rice, 1971]. The complementary potential is the Gibbs free energy, so that :

$$
W^{G}=W^{G}(\boldsymbol{\sigma}, T, \xi)=\boldsymbol{\sigma}: \boldsymbol{\epsilon}-W^{H}
$$

and

$$
\boldsymbol{\epsilon}=\frac{\partial W^{G}(\boldsymbol{\sigma}, T, \xi)}{\partial \boldsymbol{\sigma}}
$$

Variations in state at fixed $\xi$, noted $\delta \epsilon, \delta \Psi$, etc., should be reversible (path independent). More general variations that involve a change $d \xi$ are defined by $d \epsilon, d \Psi$, etc... In particular, let $d \Psi^{i}$ denote the change in the free energy function when the solid undergoes deformation that takes it from state $\xi$ to state $\xi+d \xi$ at constant $\sigma$ and $T$ :

$$
d W^{G_{i}}=W^{G}(\boldsymbol{\sigma}, T, \xi+d \xi)-W^{G}(\boldsymbol{\sigma}, T, \xi)
$$

Therefore the inelastic strain associated with $d \xi$, by differentiating equation 7 , is given by:

$$
d^{i} \epsilon_{i j}=\frac{\partial}{\partial \sigma_{i j}}\left(d W^{G_{i}}\right)
$$

Thus, inelastic variations in the potentials are themselves potentials for inelastic variations in stress and strain [Rice, 1971]. As for the full strain increment, we can write that:

$$
d \epsilon_{i j}=M_{i j k l} d \sigma_{k l}+\alpha_{i j} d T+d^{i} \epsilon_{i j}
$$


the first term corresponding to the purely elastic strain and the second to the thermoelastic effect, with the compliance tensor $M$ given by:

$$
M_{i j k l}=\frac{\partial^{2} W^{G}(\boldsymbol{\sigma}, T, \xi)}{\partial \sigma_{i j} \partial \sigma_{k l}}
$$

Hence, following this framework, we can develop a damage-constitutive model that accounts for inelastic deformation. Next section (2.2) defines the internal variable $(\xi)$ used in our micromechanical model to describe the inelastic state, and then using the energy-based approach, we develop a constitutive strain-stress relationship (section 2.5).

\subsection{Inelastic deformation in the brittle crust is largely controlled by the presence of pre-existing fractures.}

Inelastic deformation in the brittle crust occurs by nucleation, growth and/or sliding on pre-existing "fractures" at different scales. Fractures includes faults and joints but also smaller-scale cracks and flaws such as, mineral twins or defects in the crystal structure, grainboundaries and pores. Frictional sliding occurs under compressive stress on pre-existing fractures when the shear stress overcomes the frictional resistance acting on the fracture interface. Tensile cracking on the other hand can have different origins. Under regional tensile loading, the stress concentration allows the local stresses at the cracks tips to exceed the rock strength, leading to crack propagation. Tensile cracking can also occur by hydraulic cracking: locally the pore pressure increase can lead to tensile stresses at the crack tips, even under compressive loading. Finally, frictional sliding on fractures under compression creates a tensile force (as the faces slide in opposing direction) that opens wing cracks at the tip of the shear fractures. The wing cracks nucleate and grow in $\sigma_{1}$ direction (most compressive) and open in $\sigma_{3}$ direction (least compressive).

Flaws described above (e.g., fractures, microcracks, faults, mineral defects, grain-boundaries, pores), are found in all natural rocks. Therefore, for the purpose of this study, and following Ashby and Sammis [1990], Deshpande and Evans [2008], we represent the medium surrounding faults as an isotropic elastic solid that contains pre-existing monosized flaws, here represented by penny-shaped cracks of radius $a$ (Figure 1). They are assumed to have a volume density $N_{v}$ (prescribed) that remains fixed during the loading (i.e., no nucleation of new cracks). The density of these initial flaws per unit volume are characterized by a scalar $D_{0}$ defined as:

$$
D_{0}=\frac{4 \pi}{3} N_{v}(\alpha a)^{3}
$$

where $\alpha a$ is the projection of the crack radius parallel to the direction of $\sigma_{1}$. We only take into account the cracks that are optimally oriented from a Coulomb friction perspective for a given stress state, i.e., the cracks are aligned at the same angle $\Phi$ to the largest (most negative) remote compressive stress, $\sigma_{1}$ (see Bhat et al. [2011] for a justification):

$$
\Phi=\frac{1}{2} \tan ^{-1}(1 / f)
$$

where $f$ is the coefficient of friction.

Under suitable conditions, inelastic deformation occurs in the model by either opening the pre-existing cracks or by propagation of cracks. For simplicity, we account for opening and propagation of cracks due to regional tensile loading and frictional sliding (compressive loading) but we do not include hydrofracturing in the model. Cracks grow in the form of tensile wing cracks that nucleate at the tips of the penny shaped flaws. Wings cracks, each of length $l$, grow parallel to the $\sigma_{1}$ axis (Figure 1) and then, the current damage state is defined by the scalar $D$, (fraction of volume occupied by micro-cracks):

$$
D=\frac{4 \pi}{3} N_{v}(\alpha a+l)^{3}
$$


$D$ approaching 1, corresponds to the coalescence stage that leads to the macroscopic fracture of the solid. Henceforth, the internal variable $D$, that describes the current damage state of our solid, denotes the inelastic state of the material (replaces $\xi$ in section 2.1).

\subsection{Gibbs free energy of a damage solid}

In this paper, we use the aforementioned energy-based framework (section 2.1) to determine the strain-stress relationship of a damaged solid by defining the constitutive relationship in terms of Gibbs free energy $W^{G}$. Henceforth, we assume isothermal conditions, and the Gibbs free energy density of damaged solid, for a given stress state $\sigma$ and damage state $D$, can be written as the sum of (1) the free energy $W^{G_{e}}(\boldsymbol{\sigma})$ of a solid, without flaws, deforming purely elastically and (2) the free energy $W^{G_{i}}(\boldsymbol{\sigma}, D)$ corresponding to the contribution of micro-cracks :

$$
W^{G}(\boldsymbol{\sigma}, D)=W^{G_{e}}(\boldsymbol{\sigma})+W^{G_{i}}(\boldsymbol{\sigma}, D)
$$

Consequently, the associated elastic and inelastic strains, $\epsilon_{i j}^{e}$ and $\epsilon_{i j}^{i}$ respectively, can be expressed as:

$$
\epsilon_{i j}=\epsilon_{i j}^{e}+\epsilon_{i j}^{i}=\frac{\partial W^{G_{e}}(\boldsymbol{\sigma})}{\partial \sigma_{i j}}+\frac{\partial W^{G_{i}}(\boldsymbol{\sigma}, D)}{\partial \sigma_{i j}}
$$

Properties of the linear elastic material are described by its shear modulus $\mu$, Poisson's ratio $\nu$, and mass density $\rho$. The elastic strain energy density is given by:

$$
W^{G_{e}}(\boldsymbol{\sigma})=\frac{1}{4 \mu}\left[2 \tau^{2}+\frac{3(1-2 \nu)}{(1+\nu)} \sigma^{2}\right]
$$

Then, since all cracks in our model have the same orientation, the Gibbs function associated with inelastic deformation at constant $\sigma$ can be written in terms of the Gibbs free energy per crack $\Delta W^{G}(\boldsymbol{\sigma}, D)$ time the number of crack per unit volume $\left(N_{v}\right)$ :

$$
W^{G_{i}}(\boldsymbol{\sigma}, D)=N_{v} \Delta W^{G}(\boldsymbol{\sigma}, D)
$$

The Gibbs free energy per crack depends on the fracture energy release rate $G$ (crack growth) and the surface energy $\gamma_{s}$ (to create a surface):

$$
\Delta W^{G}(\boldsymbol{\sigma}, D)=\int_{o}^{\Gamma}\left[G(\boldsymbol{\sigma}, D)-2 \gamma_{s}\right] \mathrm{d} s
$$

where $\mathrm{d} s$ describes the position along the micro-crack and $\Gamma$ corresponds to the locus of all crack fronts. Based on fracture mechanics, for an isotropic elastic solid, the energy release rate $G$ can be related to the stress intensity factors at the tip of the crack by:

$$
G(\boldsymbol{\sigma}, D)=\frac{1-\nu^{2}}{E}\left[K_{I}^{2}(\boldsymbol{\sigma}, D)+K_{I I}^{2}(\boldsymbol{\sigma}, D)+\frac{K_{I I I}^{2}(\boldsymbol{\sigma}, D)}{(1-\nu)}\right]
$$

Where $E$ is the Young's modulus. However, under dynamic loading rates, the wing cracks quickly quit Mode II and Mode III to become purely tensile. As a consequence their contributions are neglected in this model. We also neglect the work done by the starter flaws. The total Gibbs free energy of the damaged solid can thus be approximated as :

$$
W^{G}(\boldsymbol{\sigma}, D)=W^{G_{e}}(\boldsymbol{\sigma})+N_{v} \int_{\Gamma}\left[\frac{1-\nu^{2}}{E} K_{I}^{2}(\boldsymbol{\sigma}, D)-2 \gamma_{s}\right] \mathrm{d} s
$$

The evaluation of the Gibbs free energy, and thus the mechanical constitutive description of the modeled brittle material, is hence based on the evaluation of the stress intensity factor $K_{I}$ at the tip of the micro-cracks inside the solid (see section 2.4). 


\subsection{Evaluating the stress intensity factors at the tip of the crack.}

To account for the energy "lost" in the medium due to inelastic deformation in the brittle crust, we therefore need to compute the stress intensity factor $K_{I}$. Once the loading is sufficiently large to induce inelastic deformation, it does so by opening pre-existing cracks or by propagation of cracks. Based on structural observations (see section 2.2) we defined three regimes, depending on the overall stress-state: one for tensile loading and two for compressive loading. Under Regime I (compressive loading) stresses are not high enough to allow sliding or opening of the microcraks. Hence, the solid is assumed to behave like an isotropic linear elastic solid and therefore $W^{G_{i}}(\boldsymbol{\sigma}, D)$ is assumed to be zero. Still for compressive loading, Regime II is reached when the shear stress $\tau$ overcomes the frictional resistance $f(-\sigma)$ acting on micro-cracks. Then, has previously described, inelastic deformation is accounted for by growing tensile wing cracks at the tip of the penny shaped cracks. Finally, our model also accounts for the overall remote tensile loading (Regime III ). In that particular case, both penny shaped cracks and wing cracks open due to normal tensile stress.

Under Regime II (compressive loading), the Mode-I stress intensity factor $K_{I}^{R-I I}$ for a unit volume containing $N_{v}$ cracks of size $(l+\alpha a)$, can be expressed as :

$$
K_{I}^{R-I I}(\sigma, D)=\sqrt{\pi a_{0}}[A(D) \sigma+B(D) \tau]
$$

where $\sigma$ and $\tau$ correspond respectively to the first invariant of the stress tensor and the second invariant of the deviatoric stress tensor (equation 11 in Bhat et al. [2012]). The parameters $A$ and $B$ both depend on the damage variable, with:

$$
\begin{aligned}
& A(D)=f c_{1}(D)+c_{3}(D)\left[f c_{2}(D)+1\right] \\
& B(D)=c_{1}(D)+c_{2}(D) c_{3}(D)
\end{aligned}
$$

and

$$
\begin{aligned}
c_{1}(D) & =\frac{\sqrt{1-\alpha^{2}}}{\pi \alpha^{3 / 2}\left[\left(D / D_{0}\right)^{1 / 3}-1+\beta / \alpha\right]^{3 / 2}} \\
c_{2}(D) & =\left(\frac{\sqrt{1-\alpha^{2}}}{\alpha}\right)\left(\frac{D_{0}^{2 / 3}}{1-D^{2 / 3}}\right) \\
c_{3}(D) & =\frac{2 \sqrt{\alpha}}{\pi}\left[\left(D / D_{0}\right)^{1 / 3}-1\right]^{1 / 2}
\end{aligned}
$$

$S_{i j}$ :

Regime III prevails under tensile loading and the stress intensity factor $K_{I}^{R-I I I}$ is a quadratic function of the stress invariants:

$$
K_{I}^{R-I I I}(\sigma, D)=\sqrt{\pi a_{0}}\left[C^{2}(D) \sigma^{2}+O^{2}(D) \tau^{2}\right]^{1 / 2}
$$

with

$$
\begin{aligned}
& C(D)=A+\Omega \sqrt{\alpha\left[D / D_{0}\right]^{1 / 3}} \\
& O(D)=\sqrt{\frac{B^{2} C^{2}}{C^{2}-A^{2}}}
\end{aligned}
$$

\subsection{Determining the constitutive stress-strain relationship}

Following the energy-based approach described in sections 2.1 and 2.3, we can define the constitutive stress-strain relationship for a damage solid. The Gibbs free energy function $W^{G}(\sigma, D)$ is determined by computing the stress intensity factors $K_{I}$ as described in section 2.4, depending on the regime. Then, following equations 6 and $10, W^{G}$ is differentiated once with respect to stress to obtain the strain-stress relationship, and twice with respect to 
stress to get the compliance tensor. The Gibbs free energy can also be expressed in term of the conjugate strains invariants:

$$
\epsilon=\epsilon_{k k} \quad \text { and } \quad \gamma=\sqrt{2 e_{i j} e_{i j}} \quad \text { with } \quad e_{i j}=\epsilon_{i j}-\frac{\epsilon}{3} \delta i j
$$

which gives the Helmotz free energy, $W^{H}$ (see equation 5). Differentiating twice $W^{H}(\epsilon, D)$ with respect to strain will then give the stiffness tensor $C_{i j k l}$.

\subsubsection{Constitutive relationship for Regime I}

Under Regime I, there is no sliding or opening of the microcraks. Therefore the Gibbs free energy is given by:

$$
W^{G}(\boldsymbol{\sigma}, D)=W^{G_{e}}(\boldsymbol{\sigma})=\frac{1}{4 \mu}\left[2 \tau^{2}+\frac{3(1-2 \nu)}{(1+\nu)} \sigma^{2}\right]
$$

and the strain-stress relationship follows linear elasticity:

$$
\sigma_{i j}=2 \mu\left[\epsilon_{i j}+\frac{\nu}{1-2 \nu} \epsilon \delta_{i j}\right]=2 \mu \epsilon_{i j}+\lambda \epsilon \delta_{i j}
$$

where $\lambda$ is the Lamé's first parameter.

\subsubsection{Constitutive relationship for Regime II}

For Regime II and III, following equation 20, the Gibbs free energy can be written as the sum of the elastic contribution, $W^{G_{e}}(\boldsymbol{\sigma})$, and the inelastic contribution due to the presence of microcracks, $W^{G_{i}}(\boldsymbol{\sigma}, D)$ (see equation 20). Following Deshpande and Evans [2008] and Bhat et al. [2012], we assume that the constants $A$ and $B$ of $K_{I}^{R-I I}$ and $C$ and $O$ of $K_{I}^{R-I I I}$ are only a function of the ratio $l / a$ (and not $l$ and $a$ separately) and therefore we can treat them as constants. We thus find that the Gibbs free function for Regime II can be approximated by :

$$
W^{G}(\boldsymbol{\sigma}, D)=W^{G_{e}}(\boldsymbol{\sigma})+\frac{1}{4 \mu}\left[A_{1} \sigma+B_{1} \tau\right]^{2}
$$

where

$$
A_{1}=A \sqrt{\frac{\pi D_{0}(1-\nu)}{\alpha^{3}}} \text { and } \quad B_{1}=B \sqrt{\frac{\pi D_{0}(1-\nu)}{\alpha^{3}}} ;
$$

If we express the above expression in term of conjugate strains ( $\epsilon$ and $\gamma$ ), we obtain the Helmotz free energy that can be differentiated once with respect to strain to obtain the stress-strain relation:

$$
\begin{aligned}
\sigma_{i j}= & \frac{\mu}{\Gamma}\left\{\left(\frac{3(1-2 \nu)}{(1+\nu)}+A_{1}^{2}-\frac{A_{1} B_{1} \epsilon}{\gamma}\right) \epsilon_{i j}\right. \\
+ & \left.\left(\frac{3 \nu}{(1+\nu)}+\frac{B_{1}^{2}}{2}-\frac{A_{1}^{2}}{3}+\frac{A_{1} B_{1} \epsilon}{3 \gamma}\right) \epsilon \delta_{i j}-\left(\frac{A_{1} B_{1}}{2}\right) \gamma \delta_{i j}\right\} \\
& \text { with } \quad \Gamma=\left[\frac{3(1-2 \nu)}{2(1+\nu)}+\frac{3(1-2 \nu) B_{1}^{2}}{4(1+\nu)}+\frac{A_{1}^{2}}{2}\right]
\end{aligned}
$$

Based on equation 35 we can define the equivalent Lamé parameters $\mu^{*}$ and $\lambda^{*}$ :

$$
\mu^{*}=\frac{\mu}{2 \Gamma}\left(\frac{3(1-2 \nu)}{(1+\nu)}+A_{1}^{2}\right) \quad \text { and } \quad \lambda^{*}=\frac{\mu}{\Gamma}\left(\frac{3 \nu}{(1+\nu)}+\frac{B_{1}^{2}}{2}-\frac{A_{1}^{2}}{3}\right)
$$

and therefore approximate the change in waves speed occurring in the medium:

$$
c_{p}^{*}=\sqrt{\frac{\lambda^{*}+2 \mu^{*}}{\rho}} \text { and } c_{s}^{*}=\sqrt{\frac{\mu^{*}}{\rho}}
$$




\subsubsection{Constitutive relationship for Regime III}

Under Regime III, the Gibbs free energy is given by:

$$
W^{G}(\boldsymbol{\sigma}, D)=W^{G_{e}}+\frac{1}{4 \mu}\left[C_{1}^{2} \sigma^{2}+O_{1}^{2} \tau^{2}\right]
$$

where

$$
C_{1}=C \sqrt{\frac{\pi D_{0}(1-\nu)}{\alpha^{3}}} \text { and } O_{1}=O \sqrt{\frac{\pi D_{0}(1-\nu)}{\alpha^{3}}} ;
$$

If we express the above expression in term of conjugate strains, and differentiating the obtained Helmotz free energy, $W^{H}$, with respect to strain we can derive the the constitutive relationship:

$$
\sigma_{i j}=\mu\left\{\left(\frac{4}{2+O_{1}^{2}}\right) \epsilon_{i j}+\left(\frac{2}{\frac{3(1-2 \nu)}{(1+\nu)}+C_{1}^{2}}-\frac{4}{3\left[O_{1}^{2}+2\right]}\right) \epsilon \delta_{i j}\right\}
$$

Following the same logic as for Regime II we can define the equivalent Lamé parameters $\mu^{*}$ and $\lambda^{*}$ :

$$
\mu^{*}=\mu\left(\frac{4}{2+O_{1}^{2}}\right) \quad \text { and } \quad \lambda^{*}=\mu\left(\frac{2}{\frac{3(1-2 \nu)}{(1+\nu)}+C_{1}^{2}}-\frac{4}{3\left[O_{1}^{2}+2\right]}\right)
$$

and therefore compute the change in waves speed occurring in the medium.

\subsection{Criteria for Regime Transition}

Criteria to determine the Regimes to be applied in the model are defined based on the stress intensity factor $K_{I}$. In Regime I the stresses are not sufficient to allow inelastic deformation (sliding or opening of the microcraks). This implies $K_{I} \leq 0$ at the tip of the cracks ( $K_{I}^{R I I}$ or $K_{I}^{R I I}$, since $C$ and $O$ are related to $A$ and $B$ ). Therefore, based on equation 21 the criteria for Regime I is:

$$
A \sigma+B \tau \leq 0
$$

For the two regimes (II and III) undergoing inelastic deformation, $K_{I}$ is positive and the transition between regimes is obtained by ensuring the continuity of conjugate plastic strains $\epsilon^{i}$ and $\gamma^{i}$. Following equation 8 , the conjugates plastic strains are derived as $\epsilon^{i}=\partial W^{G_{i}} / \partial \sigma$ and $\gamma^{i}=\partial W^{G_{i}} / \partial \tau$. The first invariant of the plastic strain tensor corresponds to the opening of the microcracks, whereas the second invariant is related to the frictional sliding of the pennyshaped cracks. Under compressive loading, tensile deformation only occurs by opening of the wing cracks, whereas under Regime III, both penny shaped cracks and wing cracks opened due to normal tensile stress. As a consequence $\epsilon^{i}$ is smaller for compressive loading (Regime II) than for tensile loading (Regime III). Therefore, we are in Regime II when:

$$
A \sigma+B \tau>0 \quad \text { and } \quad\left(A^{2}-C^{2}\right) \sigma+A B \tau>0
$$

and Regime III is reached for:

$$
A \sigma+B \tau>0 \quad \text { and } \quad\left(A^{2}-C^{2}\right) \sigma+A B \tau<0
$$

\subsection{Dynamic crack growth law}

In the previous sections, we have developed the constitutive laws that prescribe the response of a damaged solid to a remote tensile or compressive loading. The energy-based approach used in this model requires to compute the Gibbs free energy, $W^{G}(\boldsymbol{\sigma}, D)$, which depends on the stress tensor and the microcrack density $D$ per unit volume (section 2.3). Therefore, we need to define the state of cracks in the medium, or how they respond to remote 
loading, since as cracks grow, the state parameter D also increases (equation 13), which in turn effects the constitutive response of the material (section 2.5). To complete the constitutive model, we thus define a state evolution law for the parameter $D$. Differentiating equation 13 with respect to time, leads to:

$$
\frac{\mathrm{d} D}{\mathrm{~d} t}=\left(\frac{3 D^{2 / 3} D_{0}^{1 / 3}}{\alpha a}\right) \frac{\mathrm{d} l}{\mathrm{~d} t}
$$

where $\mathrm{d} l / \mathrm{d} t \equiv v$ corresponds to the instantaneous wing-crack tip speed. We adopt the crack growth law developed by Bhat et al. [2012] that accounts for loading rate dependent fracture initiation toughness, $K_{I C}^{D}$ [Wang et al., 2010, 2011; Zhang and Zhao, 2013] ,and propagation toughness, $K_{I}^{d}$ [Chen et al., 2009; Dai et al., 2010, 2011; Zhou and Aydin, 2010; Gao et al., 2015].

\section{Numerical Method and model description}

\subsection{Numerical method}

This study aims to evaluate the influence of the dynamic evolution of damage in the surrounding medium on seismic ruptures. Therefore the constitutive damage model described above has been implemented in the 2-D spectral element code, SEM2DPACK [Ampuero, 2012, available at https://sourceforge.net/projects/sem2d/]. Reactivation of damage depends on the state of stress in the medium, which in turn is influenced by the dynamic evolution of damage density (see section 2.5). Hence, to realize the micromechanics based model, in the context of dynamic rupture, we developed a constitutive update scheme that takes into account this intricate feedback, using a Runge-Kutta-Fehlberg (RKF) method to integrate equation 46. Between each time steps during the simulations, for a given a strain field $(\boldsymbol{\epsilon})$, we solve for the new damage density field $D$ using a RKF update. Then, given this new value of the state parameter, we solve for the stress field $(\sigma)$ using the damage constitutive law (section 2.5).

\subsection{Model set-up}

In our simulations we consider a 2-D inplane model, with a 1-D right lateral fault embedded in a brittle off-fault medium that allows for dynamic evolution of elastic moduli (Figure 1). To simplify the problem, we assume plane strain conditions. In our simulations, the medium is loaded by uniform background stresses. The maximum compressive stress $\sigma_{1}$, and the minimum compressive stress $\sigma_{3}$ are in the $x-z$ plane, whereas the intermediate principal stress $\sigma_{2}$ coincides with $\sigma_{y y}$. The fault plane makes a $60^{\circ}$ angle with $\sigma_{1}$, and we assume a uniform normal stress $\left(\sigma_{0}=\sigma_{z z}^{0}\right)$ and shear stress $\left(\tau_{0}=\sigma_{x z}^{0}\right)$ distribution on the fault, except for the nucleation-prone patch (thick grey line in Figure 1) for which we assign a value slightly above the nominal static strength (section 3.3). Finally, to warrant any interference with the propagating dynamic rupture we set the domain $(5.1 \times 18 \mathrm{~km})$ large enough and we applied absorbing boundary conditions on the edge of the computation domain. Reference values for the different parameters are summarized in Table 2.

\subsection{Friction law and nucleation procedure}

Rupture propagation along the fault plane is governed by a slip-weakening friction law [e.g. Palmer and Rice, 1973]. Slip occurs when the on-fault shear stress reaches the shear strength $\tau=f\left(-\sigma^{*}\right)$ (see section 3.4 for a definition of $\sigma^{*}$ ). The friction coefficient $f$ depends on the cumulated slip $(\delta)$ and drops from a static $\left(f_{s}\right)$ to a dynamic $\left(f_{d}\right)$ value over a characteristic distance $\delta_{c}$ :

$$
f= \begin{cases}f_{s}-\left(f_{s}-f_{d}\right) \delta / \delta_{c} & \text { if } \delta \leq \delta_{c} \\ f_{d} & \text { if } \delta>\delta_{c}\end{cases}
$$

In our models, we set the static friction coefficient at 0.6 , which corresponds to a value measured in laboratory experiments for a large range of rocks [Byerlee, 1978]. Then, follow- 


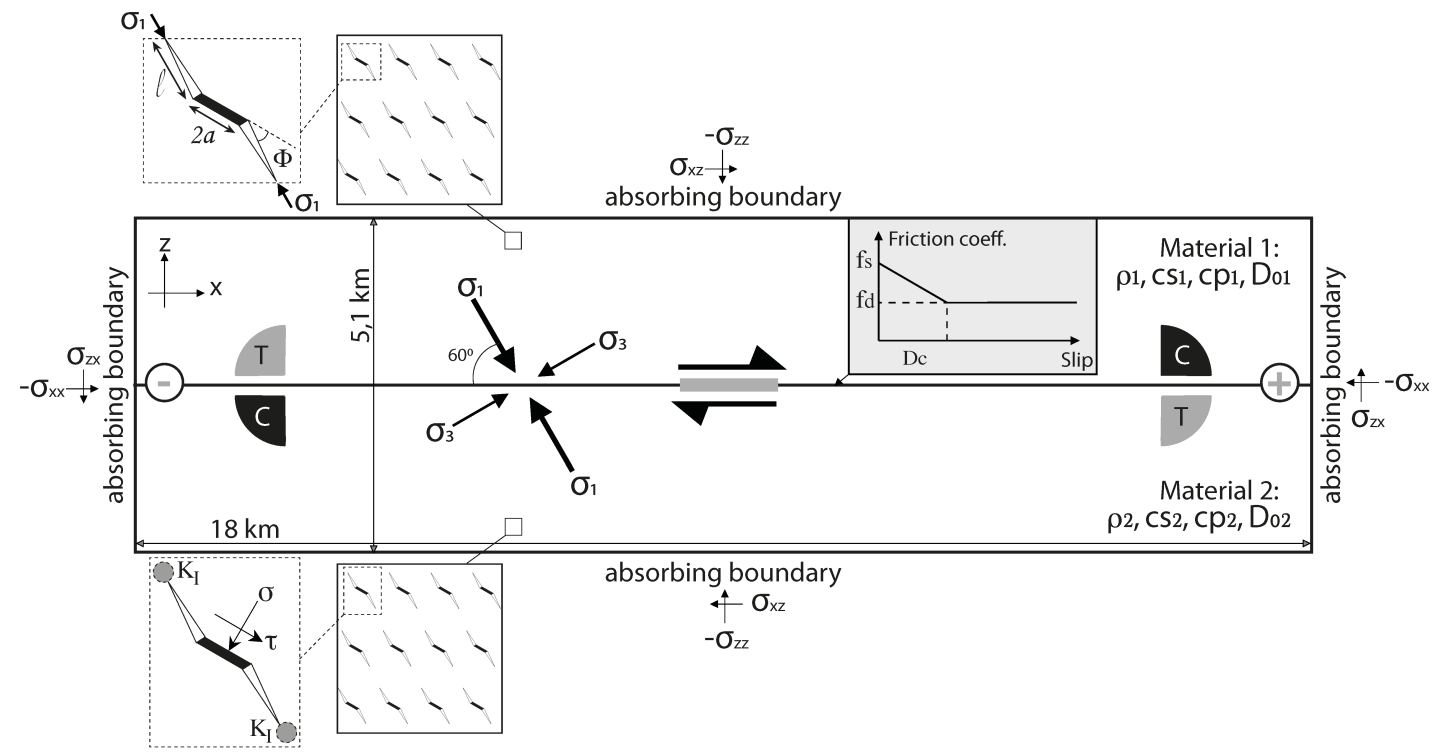

Figure 1. Schematics and parameters for simulations of dynamic ruptures in a 2-D inplane model. We consider a right-lateral planar fault (black line in the middle), embedded in a brittle off-fault medium with a damage-constitutive law. Material properties are defined by the density $(\rho)$, the $\mathrm{S}$ and P-waves speed $\left(c_{s}\right.$ and $c_{p}$ ) and the initial damage density $\left(D_{0}\right)$. In some simulations, we assume a material contrast across the fault. In that case, medium 1 is always the softer material, and material 2 the stiffer. Slip-weakening friction (grey box) acts on the $18 \mathrm{~km}$ long fault, $C$ and $T$ denoting the compressional and the tensional quadrants, respectively. Also shown are the ' + ' and '-' directions defined by the material contrast across the fault. The ' + ' direction is defined as the direction of motion of the more compliant material (M2 in our case). The medium is loaded by uniform background stresses with the maximum compressive stress $\sigma_{1}$ making an angle of $60^{\circ}$ with the fault plane. The thick grey line corresponds to the nucleation prone-patch where the initial shear stress is set-up to be just above the fault strength.

ing high strain-rate experiments at range covering earthquake slip rate, we assign a value of 0.1 for the dynamic friction coefficient [Wibberley et al., 2008].

To promote dynamic rupture we create nucleation-prone patch, in the middle of the fault (see blue line in Figure 1) for which $\tau_{0}$ is defined to be just above the fault strength $(\sim 0.03 \%$ greater). Following Kame et al. [2003], the minimum nucleation size $L_{c}$ determined by the energy balanced for a slip weakening law is :

$$
L_{c}=\frac{16}{3 \pi} \frac{\mu G}{\left(\tau^{0}-\tau_{d}\right)^{2}}=\frac{64}{9 \pi^{2}}\left(\frac{\tau_{s}-\tau_{d}}{\tau^{0}-\tau_{d}}\right)^{2}
$$

where

$$
\tau_{s}=f_{s}\left(-\sigma_{z z}\right) \text { and } \tau_{d}=f_{d}\left(-\sigma_{z z}\right)
$$

Here $\mu$ is the shear modulus, $G$, the fracture energy of the medium and $R_{0}=(3 \pi / 4)\left[\mu G /\left(\tau_{d}-\right.\right.$ $\left.\tau^{0}\right)^{2}$ ] is the length of the static sleep weakening zone as defined by Palmer and Rice [1973]. For our simulations and choice of parameters we set the size of the nucleation patch to be 1.5 $\mathrm{km}$.

\subsection{Regularization for bimaterial effect}

Andrews and Ben-Zion [1997], Cochard and Rice [2000] have shown that the problem of slip on bimaterial fault is ill-posed. Yet, in our simulations, evolution of off-fault damage 


\begin{tabular}{|c|c|c|c|}
\hline Parameter & Symbol & Value & \\
\hline normal stress on the fault $(\mathrm{MPa})$ & $\sigma_{0}$ & 60.7 & \\
\hline shear stress on the fault (MPa) & $\tau_{0}$ & 19.9 or 36.4 & \\
\hline static friction coefficient & $f_{s}$ & 0.6 & \\
\hline dynamic friction coefficient & $f_{d}$ & 0.1 & \\
\hline characteristic slip (m) & $\delta_{c}$ & 1 & \\
\hline Prakash and Clifton [1993] time (s) & $t^{*}$ & $40 \times 10^{-3}$ & \\
\hline Poisson's ratio & $\nu$ & 0.276 & \\
\hline branching speed $\left(\mathrm{km} . \mathrm{s}^{-1}\right)$ & $v_{m}$ & 1.58 & \\
\hline quasi-static fracture toughness & $K_{I C}^{s s}$ & $1.2 \times 10^{6}$ & \\
\hline Ashby and Sammis [1990] factor & $\beta$ & 0.1 & \\
\hline crack factor & $\Omega$ & 2.0 & \\
\hline Parameter for material & Symbol & Granite $(\mathrm{m} 1, \mathrm{~m} 2)$ & Gabbro (m2) \\
\hline penny shape cracks radius $(\mathrm{m})$ & $a_{0}$ & 60 or $\sim 0$ & 60 \\
\hline volume density of cracks $\left(\times 10^{-7} \# / \mathrm{m}^{3}\right)$ & $N_{v}$ & 1.68 or 3.36 & 1.68 \\
\hline density & $\rho$ & $2.7 \times 10^{3}$ & $3 \times 10^{3}$ \\
\hline S-wave speed (m.s $\left.{ }^{-1}\right)$ & $c_{s}$ & $3.12 \times 10^{3}$ & $3.25 \times 10^{3}$ \\
\hline P-wave speed $\left({\left.\mathrm{m} . \mathrm{s}^{-1}\right)}^{-1}\right.$ & $c_{p}$ & $5.6 \times 10^{3}$ & $5.84 \times 10^{3}$ \\
\hline
\end{tabular}

Table 2. Input Parameters for our simulations

during the rupture leads to dynamic changes of elastic moduli, which creates a damage-related material contrast across the fault. Moreover, the effect of off-fault damage on dynamic rupture has been explored for both, homogeneous and dissimilar material. To provide a regularization to the ill-posed problem in such scenarios, a characteristic time or slip scale of normal stress response has been proposed [Cochard and Rice, 2000; Ranjith and Rice, 2001]. Following Rubin and Ampuero [2007], we adopt a simplified form of Prakash and Clifton [1993] law, where the fault strength is assumed to be proportional to a modified normal stress $\sigma^{*}$, which evolves toward a residual value over a time scale $t^{*}$, in response to abrupt change of the actual fault normal stress:

$$
\dot{\sigma}^{*}=\frac{1}{t^{*}}\left(\sigma-\sigma^{*}\right)
$$

Ideally $t^{*}$ should be much larger than the time step during the simulation $(\Delta t)$, yet much smaller that the time to undergo slip weakening, $T$. Here we use $t^{*}=4 \Delta x / c_{s} \simeq 40 \times 10^{-3} \mathrm{~s}$.

Another possibility to reduce numerical oscillations, is to add an artificial Kelvin-Voigt visco-elastic layer around the fault [e.g., Brietzke and Ben-Zion, 2006; Xu et al., 2012, 2014]. However, this may also remove true small scale features and modify the response the off-fault medium by absorbing energy. Therefore, we did not damp the high-frequency numerical noise with such method, to avoid tampering the physical response in our models.

\subsection{Resolution}

To properly solve the problem at hand, we need to define a grid spacing $\Delta x$ that is small enough to resolve the smallest physical length scale. The spatial discretization $\Delta x$ (distance between two neighbor nodes) is taken so that there are multiple cells to resolve the process zone $\Lambda$ for a slip-weakening law, the shortest wavelength $\lambda_{\min }$ and the nucleation stage.

Following Day et al. [2005], if we assume that the process zone $\Lambda$ is small enough to use small-scale yielding limit of fracture mechanics [Rice, 1968] (stress field around $\Lambda$ dominated by the singular part of the crack front) and if we assume the crack propagation to be steady, 
for a slip weakening law $\Lambda$ can be expressed as:

$$
\begin{aligned}
\Lambda & =\Lambda_{0} \varphi^{-1}\left(v_{r}\right) \\
\text { with } \quad \Lambda_{0} & =\frac{9 \pi}{32(1-\nu)} \frac{\delta_{c} \rho c_{s}^{2}}{\left(f_{d}-f_{s}\right) \sigma_{z z}}
\end{aligned}
$$

where $\Lambda_{0}$ is the static value of the process zone $\left(v_{r}=0\right)$ for a mode II rupture and $\varphi^{-1}$ is a monotonic function of the rupture speed (see Day et al. [2005] for details). Consequently, values for the process zone go from $\Lambda_{0}$ to 0 , when $v_{r}$ reaches the limiting speed, which corresponds to the Rayleigh wave speed $c_{R}$ for a mode-II rupture in homogeneous solid, and the shear-wave speed for mode III (see Rubin and Ampuero [2007] for an estimate of the $\Lambda$ when there is a material contrast across the fault). $\Lambda_{0}$ is therefore a convenient upper bound for the process zone size and numerical simulation should resolve with more that one spatial element. In our models, to ensure a good resolution for the dynamic phenomena, the domain is discretized into square $600 \times 170$ elements with 3 Gauss-Lobatto-Legendre nodes (Ngll) nonuniformly distributed per element edge. This provides an element size $h$ of $30 \mathrm{~m}$, and a $\Delta x$ of $\sim 3 \mathrm{~m}$. The grid spacing $\Delta x$ is much smaller than the element size $\mathrm{h}\left(\approx \mathrm{Ngll}^{2}\right.$ times smaller $)$ since in SEM code, each element are subdivided onto a non-regular grid of $\mathrm{Ngll} \times \mathrm{Ngll}$ nodes. Consequently, the process zone is resolved with $\sim 35$ spatial element, or $\sim 316$ nodes. This also satisfies Bhat et al. [2012] criteria that requires $h / a<1$ to properly account for off-fault damage evolution.

Finally, the timestep $\Delta t$ during the simulations is determined from the Courant-FriedrichsLewy (CFL) stability criterion:

$$
C F L=c_{p} \Delta t / \Delta x
$$

where $c_{p}$ is the P-wave speed. For stability, the CFL is taken to be 0.55 in all simulations.

\section{Results}

An important result of this paper is that we not only model what is happening on the fault plane but also the constitutive response of the surrounding medium to the dynamic rupture. In the following section we explore the dynamics of earthquake rupture and the associated generation of new damage, how it affects both the hosting medium and the rupture propagation, and what are the damage-related features that can be pertinent to interpret geophysical observations. To investigate the intricate feedbacks between off-fault damage generation and earthquake rupture propagation we start the study with a simple case, a $2 \mathrm{D}$ right-lateral fault inside an homogeneous medium (Granite), where damage is only occurring on one side. Then we increase complexity by first keeping an homogenous elastic medium but with different initial damage on both side. The last example presented in this study explores the combined effect of a bimaterial fault (Granite/Gabbro) and a damage evolution law. Reference values for the different parameters are summarized in Table 2.

\subsection{Effect of Damage on dynamic rupture for a single material}

To provide an element of comparison, we first discuss the results for a dynamic rupture in a homogeneous solid (typical Granite, see Table 2 for properties) with damage evolution only on the top side of the fault (material 1 in Figure $2 a$ ). The initial flaw size ( $a$, radius of penny shape cracks) was assumed to be $60 \mathrm{~m}$ for material 1 , which scale with secondary fractures that usually surrounds main faults that extend over several tens of kilometers. The volume density of cracks, $N_{v}$, was set to be $1.68 \times 10^{-7}$ which gives an initial damage density value, $D_{0}$, of 0.1 . To prevent damage to occur on the bottom side of the fault we simply assigned a very small initial flaw size for material $2\left(a=6 \times 10^{-8} \mathrm{~m}\right)$. Keeping $N_{v}$ constant, this returns of value of $D_{0} \simeq 0$. As a consequence, the medium on the bottom side of the fault is behaving in a purely elastic manner. 

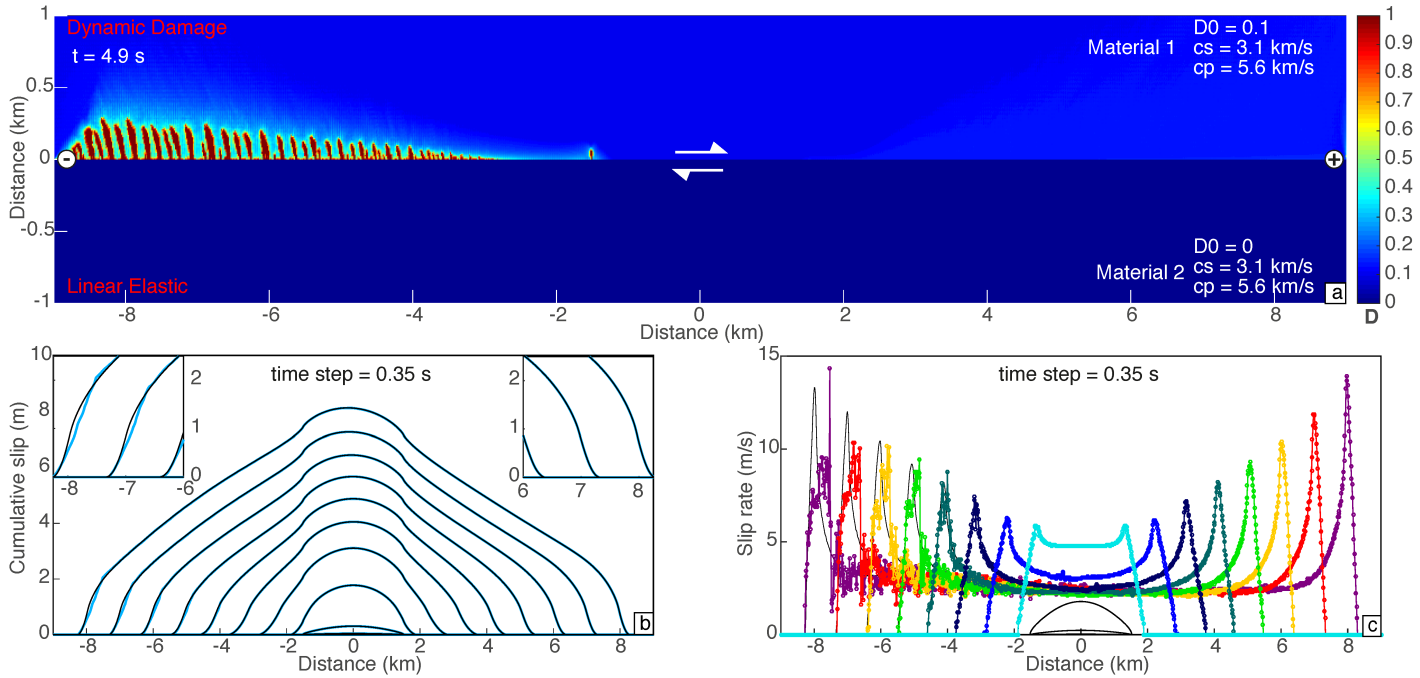

Figure 2. Simulation of a dynamic rupture on a right-lateral fault embedded in a homogeneous medium (Granite). We impose a material contrast across the fault by changing the size of the initial microcraks (60 $\mathrm{m}$ and 0 ) which leads to damage evolution only on the top side of the fault. (a) Evolution of the state parameter $D$ (density of microcracks in the medium) at $t=4.9 \mathrm{~s}$. Also shown are the '+' and '-' directions as defined in Figure 1). Dynamic damage essentially occurs in the tensile quadrant. Cumulative slip (b) and slip rate (c) on the fault are displayed with a time increment of $0.35 \mathrm{~s}$. Colored curves correspond to the dynamic simulation with the damage evolution law, thin black curves depict a simulation with the same parametrization only for a pure elastic medium.

\subsubsection{Damage density and dynamic changes of wave speeds}

Figure 2a shows a snapshot (at $t=4.9 \mathrm{~s}$ ) of the state parameter $D$ (density of microcracks in the medium), for a bilateral rupture propagating along the interface between the damaged (above in the graph) and the undamaged material (below). This corresponds to the final stage, at the end of the numerical simulation, chosen to avoid boundary effects. Time evolution of damage with respect to slip rate on the fault is also represented in Figure 3. For a right-lateral fault, the rupture tip propagating to the left puts material 1 in tension while the rupture tip on the right induce compression in the medium (T- and C-direction respectively in Figure 1). As illustrated by the parameter $D$, the response of the damaged elastic solid is different in the compressional and tensional quadrants with more damage in the tensile lobe. Thus the rupture travelling on the compressional side activates and/or interacts little with the off-fault damage whereas on the extensional side, the rupture tip induces a reduction in elastic moduli (Figure 4), which differs from a classic bimaterial rupture since the generation of damage induces a dynamic evolution of the elastic properties (or a "dynamic" bimaterial effect). Based on equations 37, 38 and 42, we record a maximum change of $32.7 \%$ for Swave and $28.0 \%$ for P-wave. Those results are consistent with geophysical observations of temporal changes in seismic velocity along natural fault following earthquake ruptures [Hiramatsu et al., 2005; Li et al., 2006; Brenguier et al., 2008; Cochran et al., 2009; Froment et al., 2014]. At the maximum, the width of the newly-created damage zone reaches $900 \mathrm{~m}$, and corresponds to the location where the higher slip rate has been recorded (Figure 2c). However, the extent of the highly damaged zone $(D \geq 0.5)$ does not exceed $300 \mathrm{~m}$.

In all our models, we also note the formation of localized damage zones which is a direct consequence of the constitutive law. This localization of high damage density could be related to the occurrence of branched faults along mature faults. On average, they makes a $60^{\circ}$ angle with the main fault plane. However, at this stage, caution must prevail and these results should be taken more qualitatively here. Capturing localization accurately in numerical simulations 
is impossible for constitutive laws that do not have an internal length scale. There are few ways to address this problem, and we are in the process of exploring these remediations. We therefore do not make any conclusions about spacing between branched faults or the width of these localized damage zones.
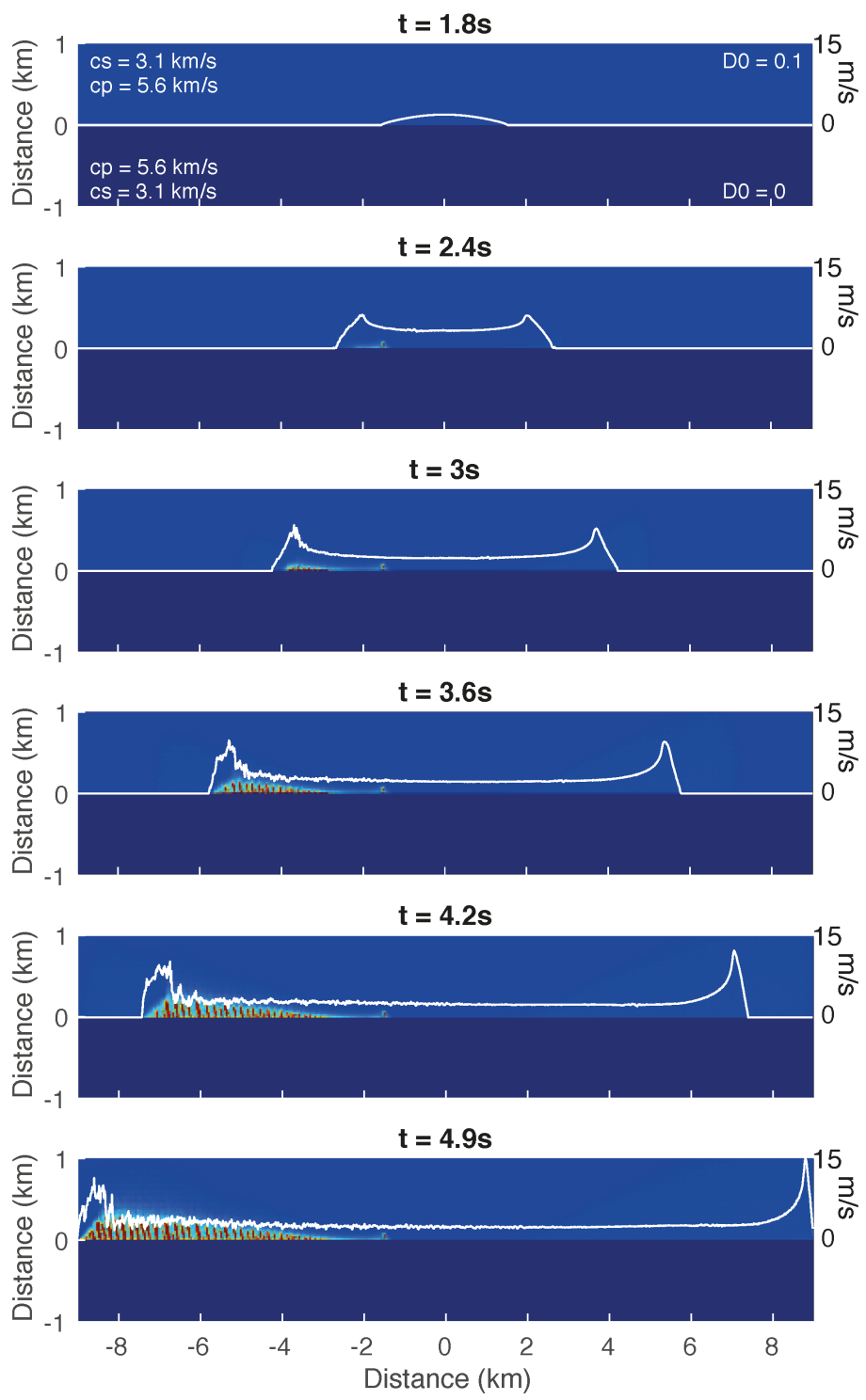

D

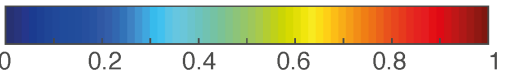

Figure 3. Temporal evolution of the damage parameter $D$ for a dynamic rupture on a right-lateral fault embedded in a homogeneous medium (Granite) with damage evolution only on the top side of the fault (see also Figure 2). Corresponding slip rate (white) is superimposed on the snapshots.

\subsubsection{Cumulative slip, slip rate and rupture speed on the fault}

Figures $2 \mathrm{~b}$ and $2 \mathrm{c}$ display respectively the cumulative slip and slip rate on the fault, with a time increment of $0.35 \mathrm{~s}$. We compare the model (colored lines) with a right-lateral rupture occurring in a pure elastic medium (thin black curves). In terms of cumulative slip, we observe 


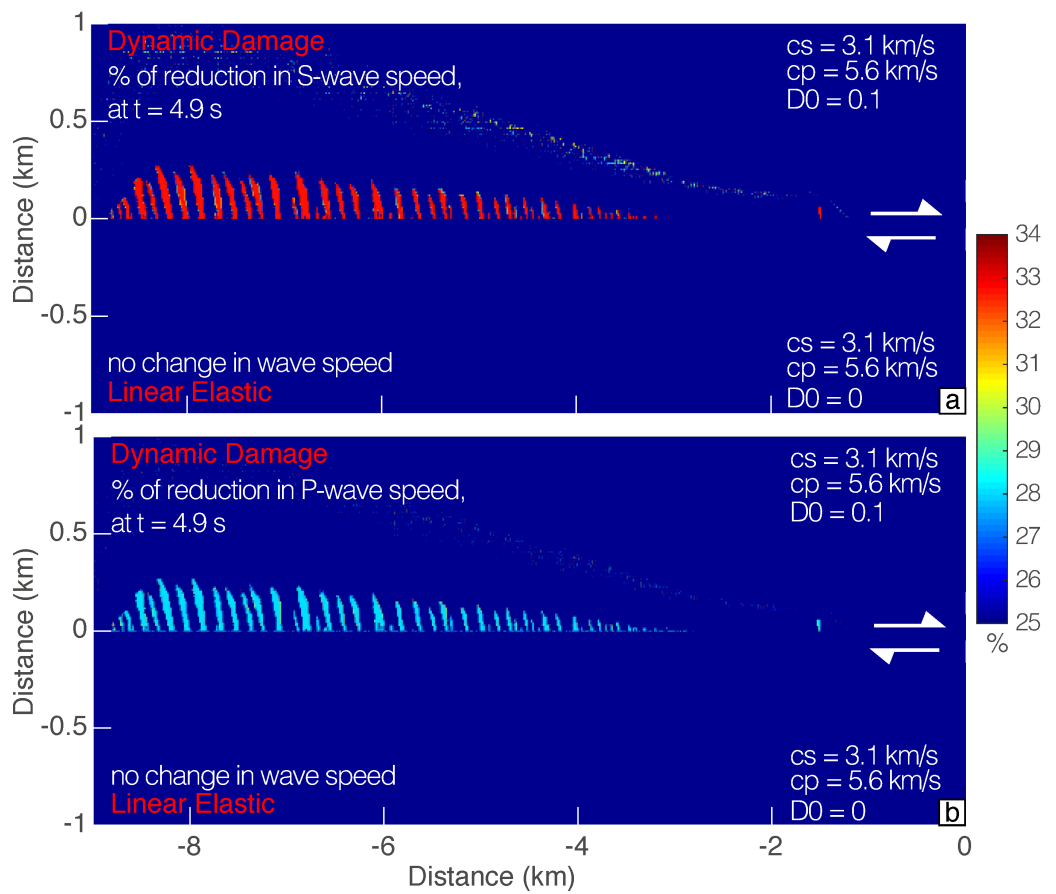

Figure 4. Reduction (in \%) of S-wave (a) and P-wave (b) speeds in the medium at $t=4.9 \mathrm{~s}$, in relation to off-fault damage for a dynamic rupture occuring in a homogeneous medium (Granite) with damage evolution only on the top side of the fault (Figure 2). Since evolution of damage essentially occurs on the upper tensile quadrant (Figure 2a) we only show that part. Formula to compute the new $c_{p}$ and $c_{s}$ values, based on the regime, are described in equations 37,38 and 42 . For this particular simulation we record a maximum change of $32.7 \%$ for S-wave speed and $28.0 \%$ for P-wave speed.

little differences with the pure elastic model. In both cases, we record a crack-like rupture and we only observe a small decrease in total slip at the rupture tip, where the widest damage zone was developed (between -8.5 and $-6 \mathrm{~km}$, left zoom in Figure $2 \mathrm{~b}$ ).

On the other hand, evolution of slip rate along the fault plane strongly differs from a classic elastic model. Figure $2 \mathrm{c}$ shows that the rupture is bilateral but is asymmetric, with more complexity in the negative direction which result in high frequency content in the radiated ground motion (see section 4.3). The development of slip rate oscillations is likely related to the development of a low velocity zone (LVZ), with up to $32.7 \%$ drop in wave-speed (Figure 4). In fact, we observe that the oscillations occur at some distance behind the rupture front and that they are developed only with the emergence of a damage zone around the fault, in relation to the rupture propagation(Figure 3). We also notice that oscillations increase in amplitude as the LVZ becomes larger. As shown by the spacing bewteen symbols on Figure 2c, that corresponds to the value at each node, the oscillations of slip rate are well resolved numerically. The development of a material contrast (LVZ) can produce internal wave reflections, which in turn gives rise to an additional feedback mechanism between the evolving off-fault medium and the dynamic rupture [e.g., Huang et al., 2014]. Similar trapped waves have been recognized during the 1992 Landers earthquake [Li et al., 1994]. From this study, authors have estimated a fault zone width of $\sim 180 \mathrm{~m}$, and a strong decrease of fault zone shear velocity $(\sim 30 \%$ ), as observed in our simulations (see in particular results for a bimaterial fault, section 4.2.2). However, in regard to the complex pattern of the LVZ, it is hard to evaluate the relative importance of the different parameters on the complicated feedbacks we can observe (e.g., velocity contrast, width and relative distance between branches, etc). 
Finally, in our simulations with damage evolution we observe little modulation of the rupture front, compared to the elastic case. This is because the dynamic rupture, which propagates at subshear velocity on average $\left(\sim 2.7 \mathrm{~km} . \mathrm{s}^{-1}\right)$, interacts with an intact material (Figure 3). However, some modulation can be observed, and sometimes the rupture can even reach supershear velocity locally. This is probably related to a process where the radiated waves interact with the LVZ behind the rupture front and further interferes with the rupture front itself.

\subsection{Evolution of damage for dissimilar materials}

For the two following scenarios we chose to increase the complexity by introducing a material contrast across the fault, as usually observed for natural cases. First, we simply keep the same material (Granite) on both sides but assign a different initial damage density (see section 4.2.1 and Table 2 for details). This could be interpreted as being the cumulative result of dynamics events, propagating dominantly in one direction, which would have created a damage contrast across the fault. In the second scenario, we assigned different elastic properties on both side, but we keep the damage density constant (see section 4.2.2 and Table 2 for details). For both scenarios, we set the initial microcrak size to be $60 \mathrm{~m}$.

\subsubsection{Variation in initial damage density}

For this particular simulation (Figure 5a), the elastic properties corresponds to those of a typical Granite (Table 2) but we change the damage density across the fault: $D_{0}=0.2$ on the top part of the fault (material 1) and $D_{0}=0.1$, for the bottom part (material 2). For an initial microcraks size of $60 \mathrm{~m}$, this leads to a volume density of cracks, $N_{v}$, of $3.36 \times 10^{-7}$ $\left(\# / m^{3}\right)$ and $1.68 \times 10^{-7}\left(\# / m^{3}\right)$, respectively.
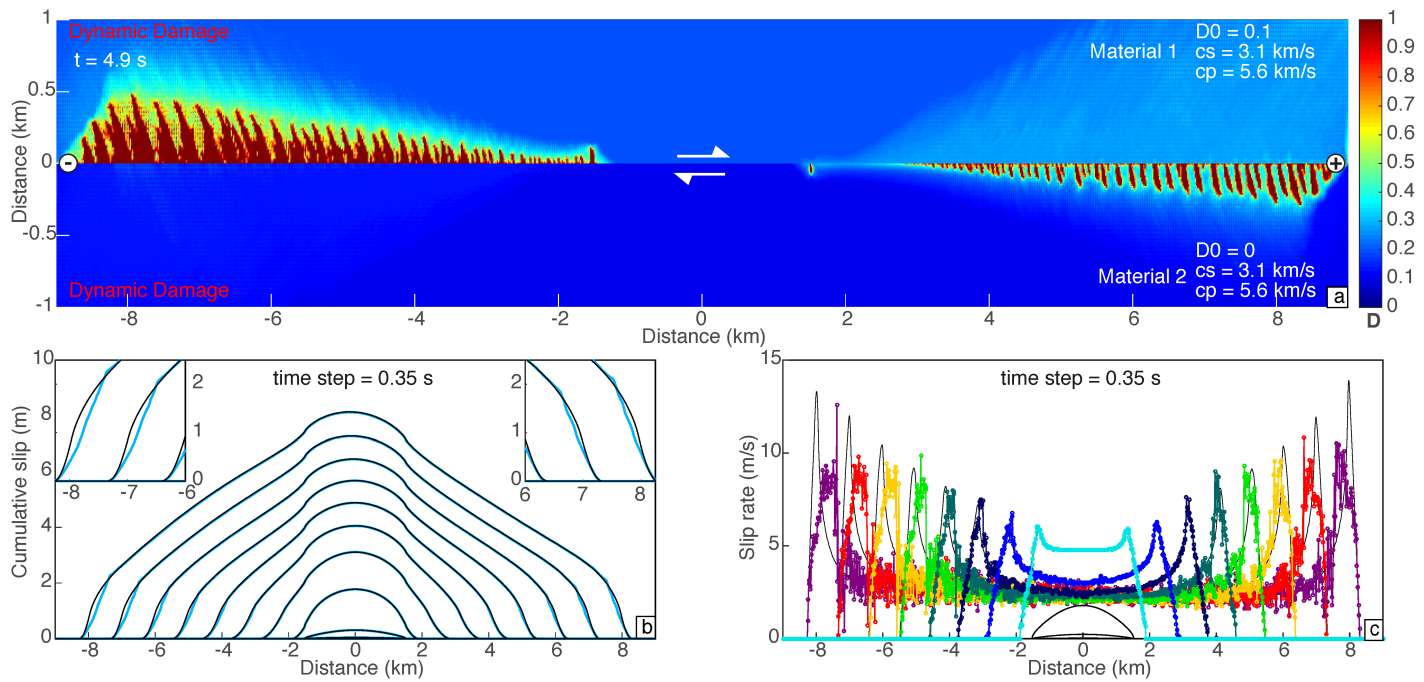

Figure 5. Simulation of a dynamic rupture on a right-lateral fault embedded in a homogeneous medium (Granite). We impose a material contrast across the fault by changing the initial damage density: $D_{0}=0.2$ on the top side of the fault, and $D_{0}=0.1$ on the bottom. (a) Evolution of the state parameter $D$ (density of microcracks in the medium) at $t=4.9 \mathrm{~s}$. The damage zone is more prominent when $D_{0}$ is higher. Also shown are the '+' and '-' directions as defined in Figure 1). Cumulative slip (b) and slip rate (c) on the fault are displayed with a time increment of $0.35 \mathrm{~s}$. Colored curves correspond to the dynamic simulation with the damage evolution law, thin black curves depict a simulation with the same parametrization only for a pure elastic medium. 
Figure 5a shows a snapshot of the state parameter $D$ at $t=4.9 \mathrm{~s}$, which corresponds to the end of the numerical simulation. Time evolution of damage with respect to slip rate on the fault is also represented in Figure 6a. The small initial difference in damage density actively impacts the final result since we observe more dynamic damage generation in the softer material $\left(D_{0}=0.2\right)$. On the left tensile quadrant, the highly damaged zone $(D \geq 0.5)$ extends up to $600 \mathrm{~m}$, whereas in the right tensile lobe, it does not exceed $300 \mathrm{~m}$. We also notice a more "gradual" decay in damage density for material 1. Concurrently, we observe a stronger reduction in elastic properties for the more compliant material (maximum change of $34.2 \%$ for S-wave and $28.2 \%$ for P-wave) than for the material with less initial damage (maximum change of $32.7 \%$ for $\mathrm{S}$-wave and $28.0 \%$ for $\mathrm{P}$-wave).

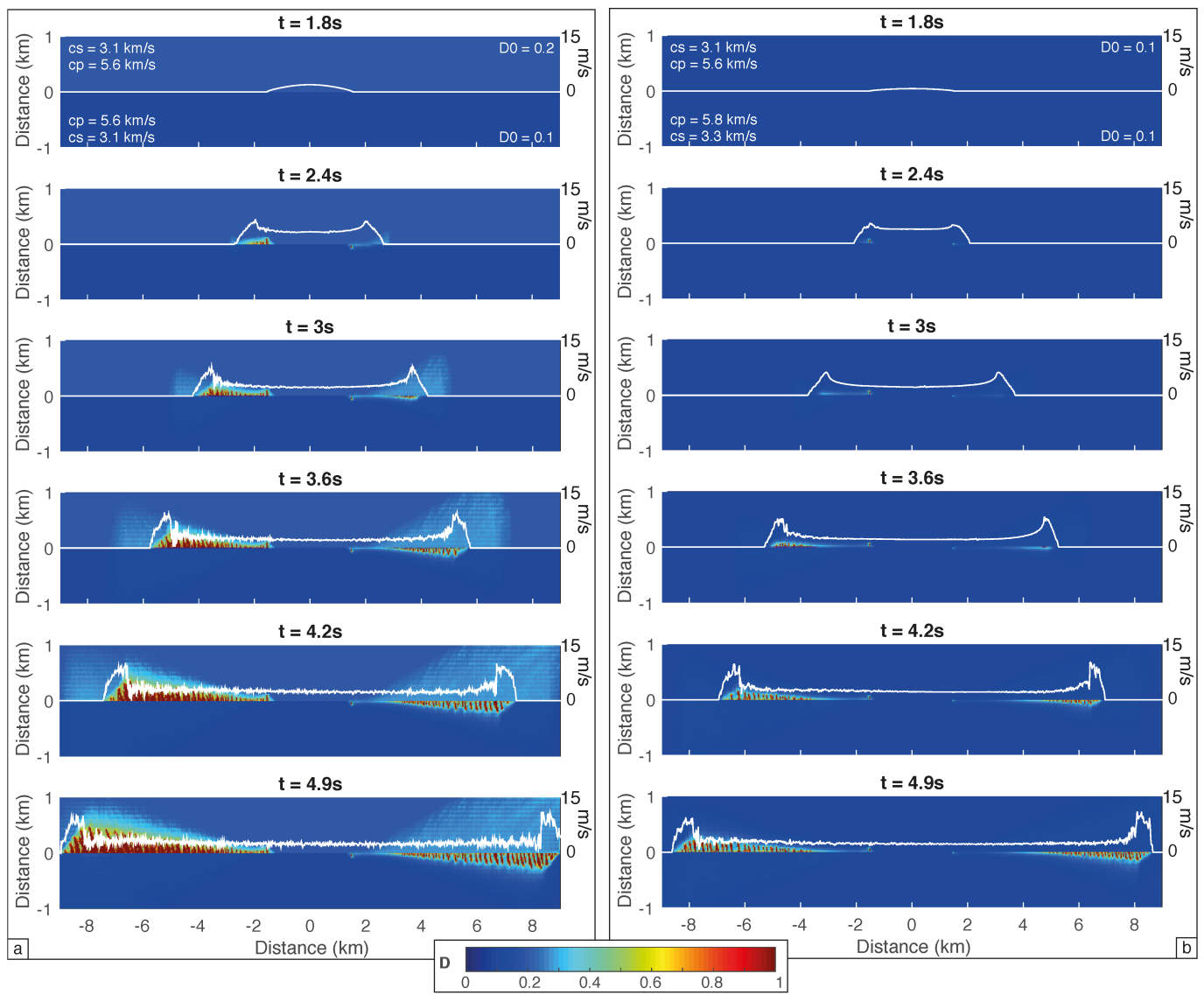

Figure 6. Temporal evolution of the damage parameter $D$ for (a) a dynamic rupture on a right-lateral fault embedded in a homogeneous medium (Granite) with a material contrast across the fault by changing the initial damage density (see also Figure 5) and (b) a dynamic rupture on a bimaterial fault with the initial damage density of $D_{0}=0.1$ on both sides. Corresponding slip rate (white) is superimposed on the snaposhots.

Figures $5 b$ and $5 c$ display respectively the cumulative slip and slip rate on the fault, with a time increment of $0.35 \mathrm{~s}$. In accordance with previous observations (section 4.1.2), the cumulative slip in the negative direction is slightly smaller than in the positive direction, where less damage is recorded. We also notice slip rate oscillations in both directions. They however occur earlier in the negative direction, in relation to the development of a LVZ that arises closer to the nucleation prone patch on that part of the fault. Finally, this simulations displays little modulation in the rupture speed, with no significant difference between the two rupture fronts. Again, this is likely related to the fact that the subshear rupture propagates inside an intact material, in both direction. 
This simple scenario underlines the importance of incorporating not only the fault history but also the off-fault medium history in dynamic modeling of earthquakes: damage can accumulate over time and influence the following rupture [Cappa et al., 2014; Huang et al., 2014]. However, one should be very cautious in developing such model since other mechanisms can kick off during the interseismic period and heal, at least partly, newly-created damage. Indeed, geophysical observations suggest that the damage effect is transient, with gradual (sometimes incomplete) recovery of the elastic properties [e.g., Brenguier et al., 2008; Froment et al., 2014]. This evolution is likely related to healing processes that affect microcracks, fractures, and faults through precipitation of soluble materials or clay mineralization [Mitchell and Faulkner, 2008].

\subsubsection{Bimaterial fault}

The third scenario explores the combined effects of a damage evolution law and a bimaterial rupture. We keep the top part of the fault (material 1) to be a Granite, whereas the bottom part properties (material 2) are typical values for Gabbro (c.f. Table 2). In both cases, we assign an initial damage density of $D_{0}=0.1$. For an initial microcraks size of $60 \mathrm{~m}$, this leads to a volume density of cracks, $N_{v}$, of $3.36 \times 10^{-7}\left(\# / \mathrm{m}^{3}\right)$.
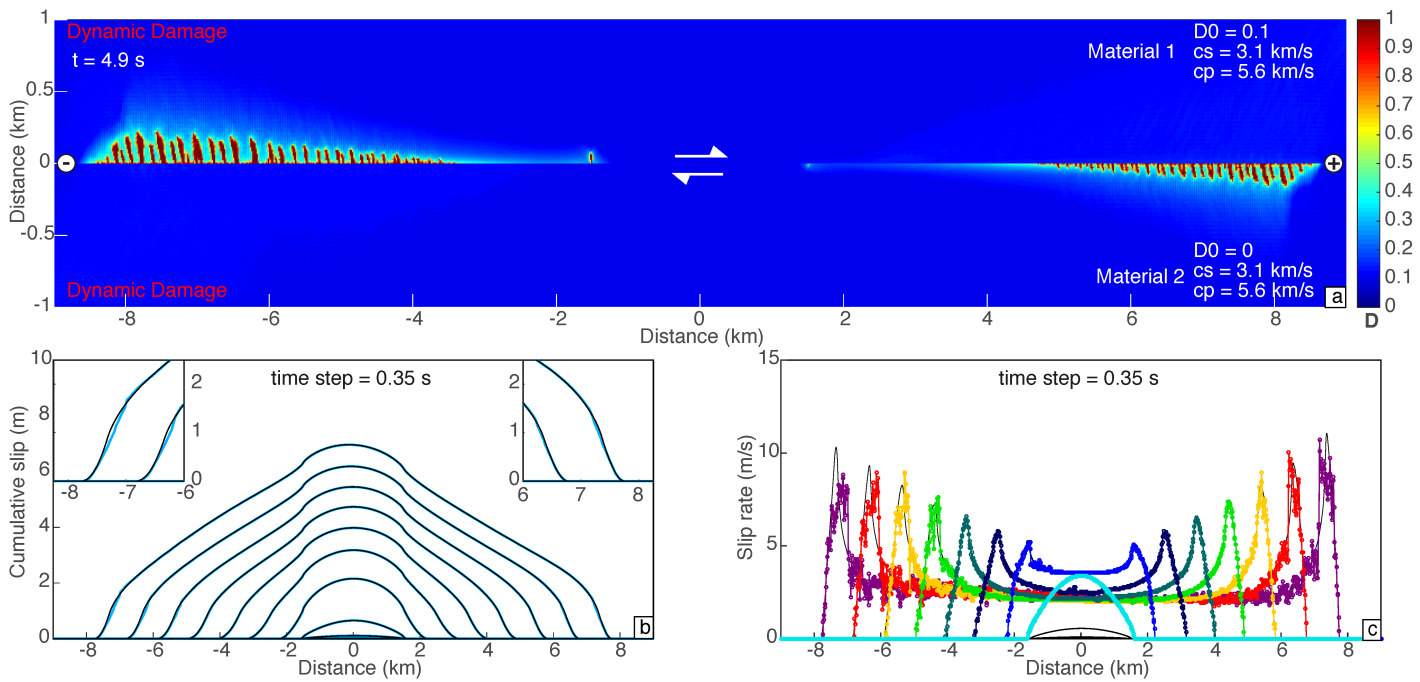

Figure 7. Simulation of a dynamic rupture on a bimaterial fault. We impose a material contrast across the fault by changing the elastic properties: Granite on the top side of the fault, and Gabbro on the bottom. In both case, we assign an initial damage density of $D_{0}=0.1$. (a) Evolution of the state parameter $D$ (density of microcracks in the medium) at $t=4.9 \mathrm{~s}$. The damage zone is more prominent for the softer material. Also shown are the ' + ' and '-' directions as defined in Figure 1). Cumulative slip (b) and slip rate (c) on the fault are displayed with a time increment of $0.35 \mathrm{~s}$. Colored curves correspond to the dynamic simulation with the damage evolution law, thin black curves depict a simulation for a bimaterial fault embedded in a pure elastic medium.

For a simulation in a homogeneous medium (same elastic properties, same $D_{0}$ ) the dynamic rupture propagates as a bilateral, symmetric, crack and produces the same damage pattern on both sides (not shown here). However, adding a material contrast across the fault (but same $D_{0}$ on both sides), creates an asymmetry in the damage pattern (Figures 7a and 6b) and the fault rupture dynamics (Figures $7 \mathrm{~b}$ and $7 \mathrm{c}$ ). For a bimaterial rupture, we observe more dynamic damage generation in the softer material. For the tensile quadrant in Granite, the highly damaged zone $(D \geq 0.5)$ extends up to $250 \mathrm{~m}$, whereas in the right tensile lobe (Gabbro), it does not exceed $160 \mathrm{~m}$. Concurrently, we observe a stronger reduction in elastic properties for the more compliant material (maximum change of $35.5 \%$ for S-wave and $31.0 \%$ 
for P-wave) than for the stiffer material (maximum change of $32.6 \%$ for S-wave and $27.9 \%$ for P-wave).

Moreover, if we compare with the homogeneous case (difference between upper parts in Figures $2 \mathrm{a}$ and $7 \mathrm{a}$ ), there is overall less damage for a bimaterial rupture. Ruptures propagating along bimaterial interface generate dynamic changes of the normal stress along the fault. For subshear ruptures, this bimaterial effect instigates a normal stress change behind the rupture tip, with a tensile stress perturbation in the positive direction and a dynamic compression in the opposite direction [e.g., Rice, 2002; Shi and Ben-Zion, 2006; Langer et al., 2013]. The effect is also sensitive to the degree of material contrast and to the details of static and dynamic friction on the fault plane. In turn, the change in normal stress influences the generation of damage. The compressive perturbation in the negative direction likely explains why the offfault medium is less damaged when the fault is a bimaterial interface.

Figures $7 \mathrm{~b}$ and $7 \mathrm{c}$ display respectively the cumulative slip and slip rate on the fault, with a time increment of $0.35 \mathrm{~s}$. The first subfigure shows a correlation between the size of the LVZ and the importance of cumulative slip reduction, in comparison to the elastic case, like we observe before. In Figure 7c, as expected, the material contrast contrast leads to a reduction in slip rate, compared to the homogeneous case (Figure $2 \mathrm{c}$ ). We also notice that the size of the damage zone correlates with the occurrence of slip rate oscillations. They are more important and occurred earlier in the negative direction, in relation to the development of a LVZ that arises closer to the nucleation prone patch on that part of the fault. As for the previous simulations, there is also very little modulation in the rupture speed, with no significant difference between the two rupture fronts (rupture propagates inside an intact material, in both direction).

In the different simulations we run, we also explore the case where we have a material and a damage density contrast across the fault (Granite \& $D_{0}=0.2$ for material 1 and, Gabbro $\& D_{0}=0.2$ for material 2). For that particular scenario (not shown here), we basically observe the combined effects described in this section and the section above. The material on the top part of the fault is even more compliant, and we observe a strong difference in damage pattern on both sides of the fault. We also notice that overall less damage is occurring than for the homogenous case (section 4.2.1). The broken symmetry in this simulation is the combined result of contrast in elastic properties and the dynamic inelastic asymmetry. The latter depends on whether the tensile or compressive stress concentration lobe is on the side of the fault with a low or high initial damage density. This is coherent with experimental studies that found that fracture damage introduces an additional asymmetry beyond that due to the associated elastic contrast [Bhat et al., 2010; Biegel et al., 2010]. Based on these simulations, one should expect to see a cumulative effect on the off-fault medium that would produce an asymmetric damage pattern across the fault, which has been observed by Dor et al. [2006].

\subsection{Effects of damage on near-fault ground motion}

This last section explores the effects of off-fault damage generation on strong ground motion in the near-source region. Figure 8 displays synthetic seismograms of fault-parallel and fault-normal velocities for a dynamic rupture on a right-lateral fault embedded in a homogeneous medium (Granite) with different initial damage on both sides of the fault (cf. section 4.2.1 and Figure 5). For comparison, we also plot the velocities for an elastic medium without damage evolution (colored curves). Seismograms are located on the extensional side and sample the two newly-created damaged zones and the medium which has not undergone any reduction in wave-speed. As expected for a sub-Rayleigh rupture, the fault-normal component dominates over the fault-parallel component in both cases. Then, if we compare the different seismograms, we observe at first that the four receivers near the fault, inside the LVZs, record long-lived signal oscillations higher in amplitude than the receivers further away from the fault. We also notice that this phenomena is even more emphasized for the two receivers located in the softer material, which has undergone a stronger reduction in elastic properties 
(section 4.2.1). Thereafter, if we compare the simulations with a damage evolution's law and those without, we can see that the velocity profiles are superimposed at first, but then there is a strong mismatch in particle velocity behind the rupture front. This is due to the fact that the rupture front propagates at first in an undamaged material. The oscillations and changes in particle velocity we further observe are related to the off-fault reduction in elastic properties due to dynamic damage and the potential reflections of seismic waves in LVZs. As a consequence, the seismograms located further away from the fault are less likely to be affected by these oscillations than the receivers inside the LVZs, which record more intense ground shaking. Seismic waves are in fact affected by the damage zone at first, but then propagate away in a homogeneous medium.

Figure 9 shows the Fourier velocity spectra of the synthetic seismograms displayed in Figure 8. For comparison, we also compute the Fourier amplitude spectra (FAS) for a dynamic rupture in an elastic medium without damage evolution (colored curves). If we compare the two models, we can see that incorporating off-fault damage evolution changes the high-frequency content of the seismograms. We observe that between 5 and $100 \mathrm{~Hz}$, Fourier velocity spectra has a higher slope than the elastic case. This contrast is essentially observed for the fault-normal component and to a smaller extent for the fault-parallel component. The difference between the two models is also more pronounced for the receivers which sample the damaged zones than for the ones further away from the fault. The complexity we observe in slip rate (section 4.1.2) together with the change in elastic properties are responsible for the high frequency content in the velocity spectra. The additional high frequency content is most likely due to the localised nature of damage. Since these localised zones are effectively cracks accelerating at a significant fraction of the shear wave speed they should contribute to the high frequency content. This is consistent with the near-fault strong motion records of real earthquakes [Housner, 1947; Wald and Heaton, 1994; Semmane et al., 2005; Dunham et al., 2011] and laboratory experiments observations. Indeed, in his $\mathrm{PhD}$ thesis, Passelegue [2015] relates the high frequency radiation recorded during laboratory earthquakes to the amount of damage that was produced. However, in natural cases, damage is not likely the only source contribution to high-frequency content. [Dunham et al., 2011] has for example demonstrated that fault-roughness induces accelerations and decelerations of the dynamic rupture, together with slip heterogeneities, which also result in ground acceleration spectra that are flat at high frequency. With the model we developed we can explore in future work these combined effects on the radiated ground motion.

\subsection{Resolution test}

We appraised the robustness of the results discussed in previous sections by comparing simulations with the same parametrization but for different grid resolutions (Figure 10). We compare simulations for a dynamic rupture on a right-lateral fault embedded in a homogeneous medium (Granite) with damage evolution only on the top side of the fault for two different mesh sizes: $30 \mathrm{~m}$ (like in section 4.1) and $15 \mathrm{~m}$. Figure 10 displays synthetic seismograms of fault-normal velocity and the corresponding FAS. Although we observe differences in the location and amplitude of the small oscillations (as a result of localization of damage), overall, the velocity profiles are farely well captured (Figure 10a to 10d). Accordingly, the Fourier analysis performed on these seismograms show very similar profiles with the same slope for the two different resolutions (Figure 10e to 10h), supporting further the robustness of the features described in section 4. Notably we observe a similar high frequency content we relate to dynamic damage generation (section 4.3), for the two different resolutions. At this stage we offer once again a note of caution that our results have to be taken more qualitatively here. As previously underlined in section 4.1.1, our constitutive law does not have an internal length scale which affects the exact localization of damage branches. 

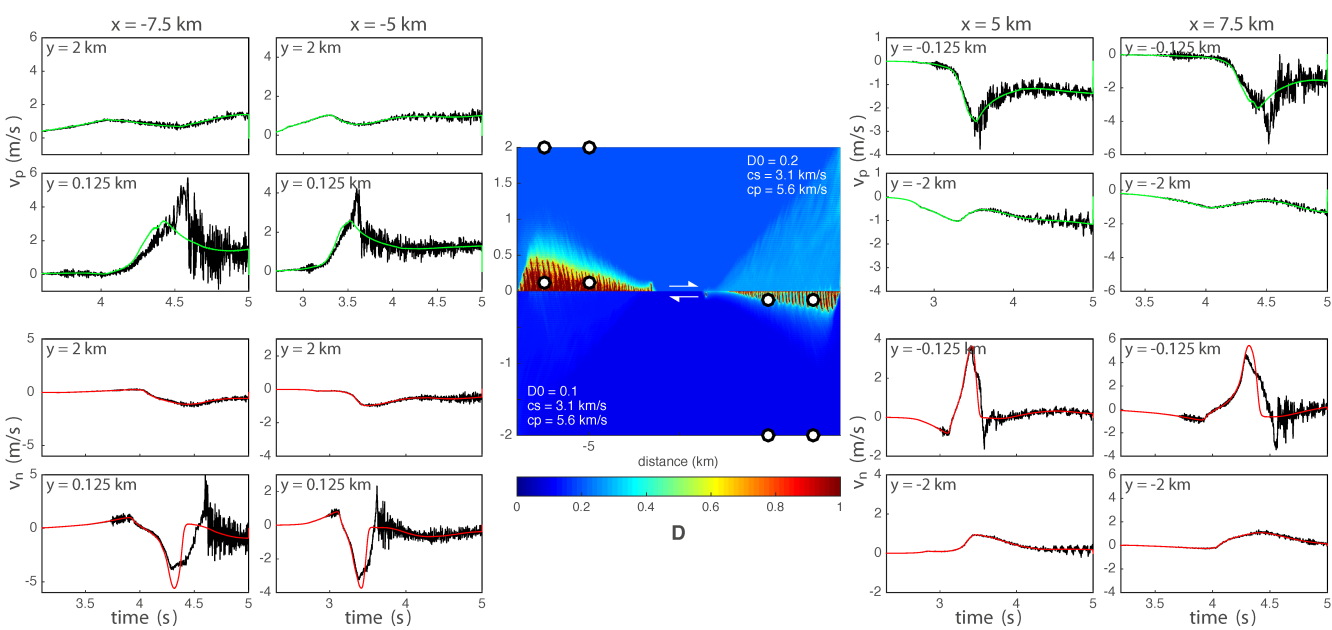

Figure 8. Synthetic seismograms of fault-parallel velocity, $v_{p}$ ( 8 upper quadrants with green curves), and fault-normal velocity, $v_{n}$ (8 lower quadrants with red curves). Seismograms are selected so that they sample the damaged and undamaged zones (see the white dots in the middle for location). Black curves correspond to the dynamic simulation with homogeneous elastic properties but different initial damage (section 4.2.1 and Figure 5). Colored curves correspond to a simulation with the same parametrization but within a pure elastic medium.
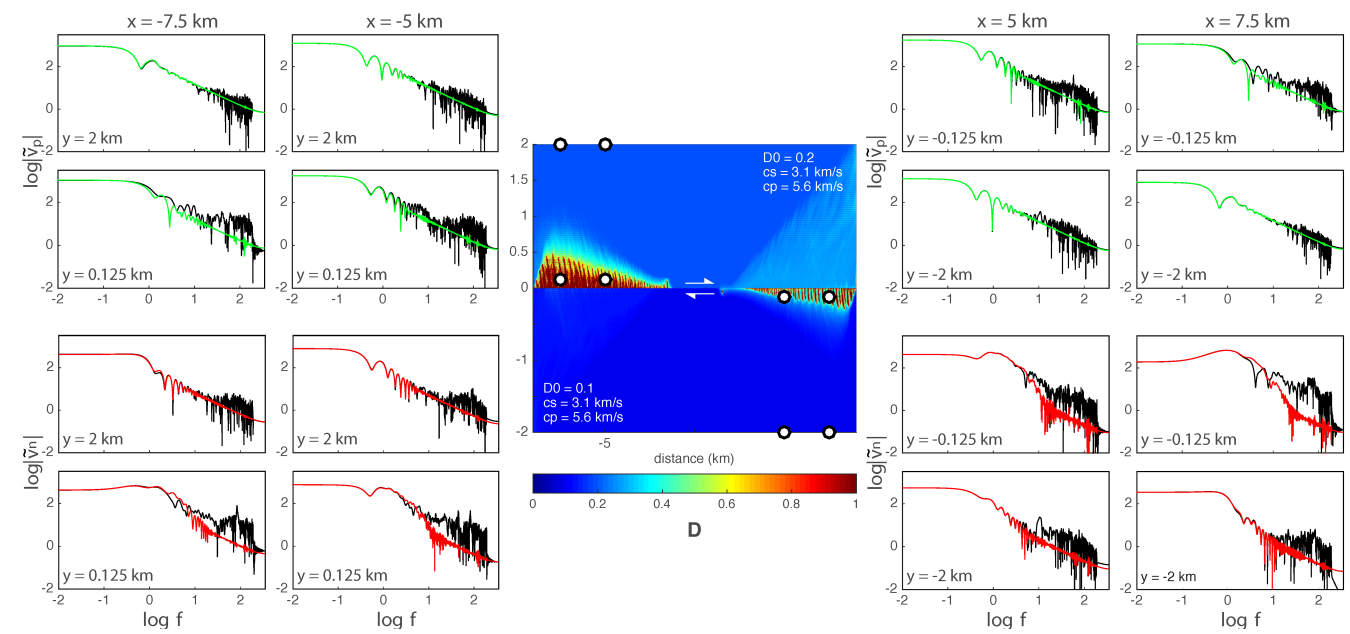

Figure 9. Fourier amplitude spectra (FAS) of fault-parallel velocity, $v_{p}(8$ upper quadrants with green curves), and fault-normal velocity, $v_{n}$ ( 8 lower quadrants with red curves), corresponding to the seismograms in Figure 8. Black curves correspond to the dynamic simulation with homogenous elastic properties but different initial damage (section 4.2.1 and Figure 5). Colored curves correspond to a simulation with the same parametrization but within a pure elastic medium. Seismograms are selected in order to sample both, the damaged and undamaged zones (see the white dots in the middle for location).

\section{Discussion and Conclusion}

In this paper, we have provided the description of a micromechanical model that accounts for the dynamic evolution of elastic properties in the surrounding medium, related to dynamic off-fault damage. We have numerically investigated the role of spontaneous off-fault damage generation on earthquake rupture processes and underlined damage-related features that can be pertinent to interpret geophysical observations. 

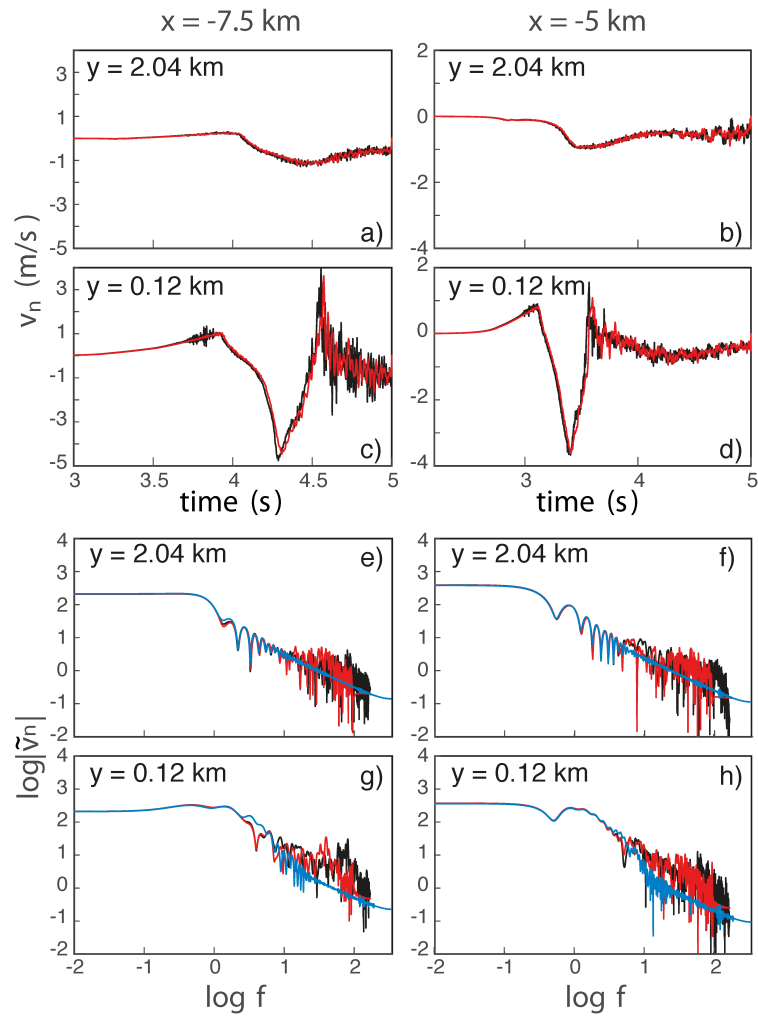

Figure 10. Numerical tests on the resolutions of our model with two different mesh sizes. We compare 4 synthetic seismograms of fault-normal velocity (a-d) and their corresponding FAS (e-h) for a dynamic rupture on a right-lateral fault embedded in a homogeneous medium (Granite) with damage evolution only on the top side of the fault (section 4.1 and Figure 2). Black and red curves correspond to the dynamic simulations with a $15 \mathrm{~m}$ and $30 \mathrm{~m}$ mesh size, respectively. For the FAS (e-h), the blue curves correspond to a simulation with a $30 \mathrm{~m}$ mesh size, with the same parametrization but within a pure elastic medium.

The main difference with models allowing for plastic deformation is that the developed constitutive law accounts for dynamic changes of elastic properties in the off-fault medium. This changes of elastic moduli, related to damage generation, has been observed along natural faults during earthquake and for laboratory experiments [e.g., Faulkner et al., 2006; Brenguier et al., 2008; Cochran et al., 2009; Froment et al., 2014]. Dynamic changes of elastic properties during earthquakes generate intricate feedbacks between the off-fault damage generation and the dynamic rupture itself, that cannot be modelled with plasticity. Moreover, in comparison with pre-existing damage models [e.g., Lyakhovsky et al., 1997b; Xu et al., 2014], our constitutive law accounts for the fracture toughness' dependency on loading rate and cracktip velocities [Chen et al., 2009; Dai et al., 2010, 2011; Wang et al., 2010, 2011; Zhang and Zhao, 2013], which is essential to model properly the micro-physics of damage evolution related to earthquake rupture.

We demonstrated that the response of the damage elastic solid is different in the compressional and tensional quadrants with more damage in the tensile lobe (section 4.1). This creates an asymmetric pattern across the fault, that is expected to increase over time if the fault ruptures in a self-similar fashion. In turn, dynamic damage influences the slip rate on the fault with the development of slip rate oscillations which result in high frequency content in the radiated ground motion.

Numerical simulation described in section 4.2.1 has underlined the importance of incorporating not only the fault history but also the off-fault medium history in dynamic modeling 
of earthquakes. A small difference in initial damage actively impacts the final pattern, with more dynamic damage generation in the softer material (greater density of initial microcracks). A significant step further, very challenging however, would be to develop numerical model of the full fault slip history that accounts for the evolution of elastic properties of surrounding medium, due to dynamic damage and healing of microcracks in the postseismic period.

In this paper, we have also explored the combined effects of having a damage evolution law and a static bimaterial fault. Detailed investigation has shown that dynamic damage generation is sensitive to material contrast and that the fracture damage introduces an additional asymmetry beyond that due to the associated elastic contrast. The asymmetry produced by the interaction between the rupture front and the off-fault damage can be opposite to that produced by velocity contrast and therefore cannot be modeled with a simple reduction in elastic stiffness. These numerical simulations are in complete agreement with previous experimental studies of mode-II ruptures on an interface that combines a bulk elastic mismatch with a contrast in off-fault damage [Bhat et al., 2010; Biegel et al., 2010].

In the last result's section we have explored the effects of off-fault damage on strong ground motion in the near-source region. The high frequency content we observe in the velocity records are related to dynamic damage generation that induces a reduction in elastic moduli, and produces slip rate oscillations on the fault. It is compatible with strong motion records of real earthquakes and laboratory experiments observations [Housner, 1947; Wald and Heaton, 1994; Semmane et al., 2005; Dunham et al., 2011; Passelegue, 2015]. These results are essential for seismic risk mitigation, however, we only investigated one possible source of high-frequency ground motion. As previously underlined by Dunham et al. [2011], fault-roughness, scattering in a non-homogeneous medium and local site conditions would also play a role in high-frequency generation during an earthquake.

Our primarily results leave open the question of the dimensionality. Obviously wave propagation should differ between a 2-D and 3-D model and therefore this would potentially affect both the damage-generation and the ground motion. However, we don't expect too much difference between 2D and 3D when it comes to rupture propagation, since most of the dynamic damage occurs behind the rupture tip. Moreover, damage is sensitive to the background stress, and one should expect to see a depth-dependency in the generation of damaged rocks, that would affect dynamic ruptures over several seismic cycles. There is clear need to extend further this kind of model in 3D.

Finally, geophysical observations suggest that off-fault damage is a transient effect since we observe a gradual recovery of the elastic properties [e.g., Froment et al., 2014]. This evolution is likely related to healing processes that affects microcracks, fractures, and faults through precipitation of soluble materials or clay mineralization [Mitchell and Faulkner, 2008]. While laboratory experiments are in favor of a relatively quick (hours to months) healing of damage [e.g., Morrow et al., 2001], field observations suggest that this damage effect can persist for several years and in some cases, for thousands of years [e.g., Cochran et al., 2009]. This means that damage simply does not cumulate over time, but it is complex competition between the intensity of the coseismic rupture, the efficiency of healing processes and time recurrence between earthquake. When it comes to model fault slip evolution over several seismic cycles, this phenomenon should be taken into account.

\section{Acknowledgments}

This study was supported by the Agence National de la Recherche (ANR) GeoSMEC contract ANR-12-BS060016. We thank the two anonymous reviewers for insightful detailed comments that helped us improve the manuscript. We also thank J.-P. Ampuero for providing the SEM2DPACK code and for his useful comments on how to add the new constitutive model to the SEM code. 


\section{References}

Ampuero, J. P. (2012), Sem2dpack, a spectral element software for 2d seismic wave propagation and earthquake source dynamics, v2.3.8 [data set], zenodo, http://doi.org/10.5281/zenodo.230363, Tech. rep., Caltech.

Andrews, D. J. (2005), Rupture dynamics with energy loss outside the slip zone, Journal of Geophysical Research-solid Earth, 110(B1), B01,307.

Andrews, D. J., and Y. Ben-Zion (1997), Wrinkle-like slip pulse on a fault between different materials, Journal of Geophysical Research-Solid Earth, 102(B1), 553-571.

Andrews, D. J., and R. A. Harris (2005), The wrinkle-like slip pulse is not important in earthquake dynamics, Geophysical Research Letters, 32(23), L23,303.

Ashby, M. F., and C. G. Sammis (1990), The damage mechanics of brittle solids in compression, Pure and Applied Geophysics, 133(3), 489-521.

Audet, P., and R. Burgmann (2014), Possible control of subduction zone slow-earthquake periodicity by silica enrichment, Nature, 510(7505), 389-392.

Ben-Zion, Y., and Z. Q. Shi (2005), Dynamic rupture on a material interface with spontaneous generation of plastic strain in the bulk, Earth and Planetary Science Letters, 236(1-2), 486496, doi:10.1016/j.eps1.2005.03.025.

Bhat, H., R. Biegel, A. Rosakis, and C. Sammis (2010), The effect of asymmetric damage on dynamic shear rupture propagation ii: With mismatch in bulk elasticity, Tectonophysics, 493(3-4), 263-271.

Bhat, H. S., C. G. Sammis, and A. J. Rosakis (2011), The micromechanics of westerley granite at large compressive loads, Pure and Applied Geophysics, 168(12), 2181-2198.

Bhat, H. S., A. J. Rosakis, and C. G. Sammis (2012), A micromechanics based constitutive model for brittle failure at high strain rates, Journal of Applied Mechanics-transactions of the Asme, 79(3), 031,016.

Biegel, R., H. Bhat, C. Sammis, and A. Rosakis (2010), The effect of asymmetric damage on dynamic shear rupture propagation i: No mismatch in bulk elasticity, Tectonophysics, 493(3-4), 254-262.

Biegel, R. L., and C. G. Sammis (2004), Relating fault mechanics to fault zone structure, in Advances in Geophysics, vol. 47, pp. 65-111, Elsevier.

Brenguier, F., M. Campillo, C. Hadziioannou, N. M. Shapiro, R. M. Nadeau, and E. Larose (2008), Postseismic relaxation along the san andreas fault at parkfield from continuous seismological observations, Science, 321(5895), 1478-1481.

Brietzke, G. B., and Y. Ben-Zion (2006), Examining tendencies of in-plane rupture to migrate to material interfaces, Geophysical Journal International, 167(2), 807-819, doi: 10.1111/j.1365-246X.2006.03137.x.

Byerlee, J. (1978), Friction of rocks, Pure and Applied Geophysics, 116(4-5), 615-626.

Cappa, F., C. Perrin, I. Manighetti, and E. Delor (2014), Off-fault long-term damage: A condition to account for generic, triangular earthquake slip profiles, Geochem. Geophys. Geosyst., 15(4), 1476-1493.

Chen, R., K. Xia, F. Dai, F. Lu, and S. Luo (2009), Determination of dynamic fracture parameters using a semi-circular bend technique in split hopkinson pressure bar testing, Engineering Fracture Mechanics, 76(9), 1268-1276.

Chester, F. M., and J. M. Logan (1986), Implications for mechanical properties of brittle faults from observations of the punchbowl fault zone, california, pure and applied geophysics, 124(1), 79-106, doi:10.1007/BF00875720.

Chester, F. M., J. P. Evans, and R. L. Biegel (1993), Internal structure and weakening mechanisms of the san andreas fault, Journal of Geophysical Research-Solid Earth, 98(B1), 771786.

Childs, C., T. Manzocchi, J. J. Walsh, C. G. Bonson, A. Nicol, and M. P. Schpfer (2009), A geometric model of fault zone and fault rock thickness variations, Journal of Structural Geology, 31(2), 117-127. 
Cochard, A., and J. R. Rice (2000), Fault rupture between dissimilar materials: Illposedness, regularization, and slip-pulse response, Journal of Geophysical Research-Solid Earth, 105(B11), 25,891-25,907.

Cochran, E. S., Y. G. Li, P. M. Shearer, S. Barbot, Y. Fialko, and J. E. Vidale (2009), Seismic and geodetic evidence for extensive, long-lived fault damage zones, Geology, 37(4), 315318.

Collettini, C., C. Viti, S. A. F. Smith, and R. E. Holdsworth (2009), Development of interconnected talc networks and weakening of continental low-angle normal faults, Geology, 37(6), 567-570.

Dai, F., R. Chen, M. Iqbal, and K. Xia (2010), Dynamic cracked chevron notched brazilian disc method for measuring rock fracture parameters, International Journal of Rock Mechanics and Mining Sciences, 47(4), 606-613.

Dai, F., K. Xia, H. Zheng, and Y. Wang (2011), Determination of dynamic rock mode-i fracture parameters using cracked chevron notched semi-circular bend specimen, Engineering Fracture Mechanics, 78(15), 2633-2644.

Dalguer, L. A., K. Irikura, and J. D. Riera (2003), Simulation of tensile crack generation by three-dimensional dynamic shear rupture propagation during an earthquake, J. Geophys. Res., 108(B3).

Day, S. M., L. A. Dalguer, N. Lapusta, and Y. Liu (2005), Comparison of finite difference and boundary integral solutions to three-dimensional spontaneous rupture, Journal of Geophysical Research-solid Earth, 110(B12), B12,307, doi:10.1029/2005JB003813.

den Hartog, S. A. M., C. J. Peach, D. A. M. de Winter, C. J. Spiers, and T. Shimamoto (2012), Frictional properties of megathrust fault gouges at low sliding velocities: New data on effects of normal stress and temperature, Journal of Structural Geology, 38, 156-171.

Deshpande, V. S., and A. G. Evans (2008), Inelastic deformation and energy dissipation in ceramics: A mechanism-based constitutive model, Journal of the Mechanics and Physics of Solids, 56(10), 3077-3100.

Dor, O., T. K. Rockwell, and Y. Ben-Zion (2006), Geological observations of damage asymmetry in the structure of the san jacinto, san andreas and punchbowl faults in southern california: A possible indicator for preferred rupture propagation direction, pure and applied geophysics, 163(2), 301-349, doi:10.1007/s00024-005-0023-9.

Dunham, E. M., D. Belanger, L. Cong, and J. E. Kozdon (2011), Earthquake ruptures with strongly rate-weakening friction and off-fault plasticity, part 2: Nonplanar faults, Bulletin of the Seismological Society of America, 101(5), 2308-2322.

Faulkner, D. R., T. M. Mitchell, D. Healy, and M. J. Heap (2006), Slip on 'weak' faults by the rotation of regional stress in the fracture damage zone, Nature, 444(7121), 922-925.

Faulkner, D. R., T. M. Mitchell, E. Jensen, and J. Cembrano (2011), Scaling of fault damage zones with displacement and the implications for fault growth processes, J. Geophys. Res., 116(B5).

Finzi, Y., E. H. Hearn, Y. Ben-Zion, and V. Lyakhovsky (2009), Structural properties and deformation patterns of evolving strike-slip faults: Numerical simulations incorporating damage rheology, Pure and Applied Geophysics, 166(10), 1537-1573, doi:10.1007/s00024009-0522-1.

Froment, B., J. J. McGuire, R. D. van der Hilst, P. Gouedard, E. C. Roland, H. Zhang, and J. A. Collins (2014), Imaging along-strike variations inmechanical properties of the gofar transform fault, east pacific rise, Journal of Geophysical Research-solid Earth, 119(9), 7175-7194.

Gabriel, A. A., J. P. Ampuero, L. A. Dalguer, and P. M. Mai (2013), Source properties of dynamic rupture pulses with off-fault plasticity, Journal of Geophysical Research-solid Earth, 118(8), 4117-4126, doi:10.1002/jgrb.50213.

Gao, G., W. Yao, K. Xia, and Z. Li (2015), Investigation of the rate dependence of fracture propagation in rocks using digital image correlation (dic) method, Engineering Fracture Mechanics, 138(Complete), 146-155, doi:10.1016/j.engfracmech.2015.02.021. 
Hill, R., and J. R. Rice (1973), Elastic potentials and structure of inelastic constitutive laws, Siam Journal On Applied Mathematics, 25(3), 448-461.

Hiramatsu, Y., H. Honma, A. Saiga, M. Furumoto, and T. Ooida (2005), Seismological evidence on characteristic time of crack healing in the shallow crust, Geophys. Res. Lett. 32(9).

Hok, S., M. Campillo, F. Cotton, P. Favreau, and I. Ionescu (2010), Off-fault plasticity favors the arrest of dynamic ruptures on strength heterogeneity: Two-dimensional cases, Geophysical Research Letters, 37, L02,306, doi:10.1029/2009GL041888.

Housner, G. W. (1947), Characteristics of strong-motion earthquakes, Bulletin of the Seismological Society of America, 37(1), 19-31.

Huang, Y., J.-P. Ampuero, and D. V. Helmberger (2014), Earthquake ruptures modulated by waves in damaged fault zones, J. Geophys. Res. Solid Earth, 119(4), 3133-3154.

Kame, N., J. R. Rice, and R. Dmowska (2003), Effects of prestress state and rupture velocity on dynamic fault branching, J. Geophys. Res., 108(B5).

Kanamori, H. (2006), Lessons from the 2004 sumatra-andaman earthquake, pp. 1927-1945, Royal Society.

Kaneko, Y., and Y. Fialko (2011), Shallow slip deficit due to large strike-slip earthquakes in dynamic rupture simulations with elasto-plastic off-fault response, Geophysical Journal International, 186(3), 1389-1403.

King, D. S. H., and C. Marone (2012), Frictional properties of olivine at high temperature with applications to the strength and dynamics of the oceanic lithosphere, Journal of Geophysical Research-solid Earth, 117, B12,203, doi:10.1029/2012JB009511.

Klinger, Y., J.-H. Choi, and A. Vallage (2016), Evolution of Fault Zone Properties and Dynamic Processes during Seismic Rupture, chap. Fault branching and long-term earthquake rupture scenario for strike-slip earthquakes, AGU monograph.

Langer, S., D. Weatherley, L. Olsen-Kettle, and Y. Finzi (2013), Stress heterogeneities in earthquake rupture experiments with material contrasts, Journal of the Mechanics and Physics of Solids, 61(3), 742-761.

Li, Y.-G., K. Aki, D. Adams, A. Hasemi, and W. H. K. Lee (1994), Seismic guided waves trapped in the fault zone of the landers, california, earthquake of 1992, J. Geophys. Res., 99(B6), 11,705-11,722.

Li, Y.-G., P. Chen, E. S. Cochran, J. E. Vidale, and T. Burdette (2006), Seismic evidence for rock damage and healing on the san andreas fault associated with the $2004 \mathrm{~m}$ 6.0 parkfield earthquake, Bulletin of the Seismological Society of America, 96(4B), S349S363, doi:10.1785/0120050803.

Lyakhovsky, V., and Y. Ben-Zion (2014), A continuum damage-breakage faulting model and solid-granular transitions, Pure and Applied Geophysics, 171(11), 3099-3123, doi: 10.1007/s00024-014-0845-4.

Lyakhovsky, V., Y. Ben-Zion, and A. Agnon (1997a), Distributed damage, faulting, and friction, Journal of Geophysical Research-solid Earth, 102(B12), 27,635-27,649.

Lyakhovsky, V., Z. Reches, R. Weinberger, and T. E. Scott (1997b), Non-linear elastic behaviour of damaged rocks, Geophysical Journal International, 130(1), 157-166.

Ma, S. (2008), A physical model for widespread near-surface and fault zone damage induced by earthquakes, Geochem. Geophys. Geosyst., 9(11).

Mitchell, T. M., and D. R. Faulkner (2008), Experimental measurements of permeability evolution during triaxial compression of initially intact crystalline rocks and implications for fluid flow in fault zones, Journal of Geophysical Research-solid Earth, 113(B11), B11,412.

Mitchell, T. M., and D. R. Faulkner (2009), The nature and origin of off-fault damage surrounding strike-slip fault zones with a wide range of displacements: A field study from the atacama fault system, northern chile, Journal of Structural Geology, 31(8), 802-816.

Morrow, C. A., D. E. Moore, and D. A. Lockner (2001), Permeability reduction in granite under hydrothermal conditions, J. Geophys. Res., 106(B12), 30,551-30,560.

Ngo, D., Y. Huang, A. Rosakis, W. A. Griffith, and D. Pollard (2012), Off-fault tensile cracks: A link between geological fault observations, lab experiments, and dynamic rupture models, 
J. Geophys. Res., 117(B1).

Niemeijer, A., C. Marone, and D. Elsworth (2010), Fabric induced weakness of tectonic faults, Geophysical Research Letters, 37, L03,304.

Palmer, A. C., and J. R. Rice (1973), The growth of slip surfaces in the progressive failure of over-consolidated clay, Proceedings of the Royal Society of London A: Mathematical, Physical and Engineering Sciences, 332(1591), 527-548, doi:10.1098/rspa.1973.0040.

Passelegue, F. (2015), Experimental study of the seismic rupture., Ph.D. thesis, Laboratoire de Gologie de lcole Normale Suprieure, advisors: Ral Madariaga and Alexandre Schubnel.

Prakash, V., and R. Clifton (1993), Time resolved dynamic friction measurements in pressure shear, Experimental Techniques in the Dynamics of Deformable Solids, 165, 33-48.

Ranjith, K., and J. Rice (2001), Slip dynamics at an interface between dissimilar materials, Journal of the Mechanics and Physics of Solids, 49(2), 341-361.

Rice, J. R. (1968), A path independent integral and the approximate analysis of strain concentration by notches and cracks, Journal of Applied Mechanics, 35(2), 379-386, doi: 10.1115/1.3601206.

Rice, J. R. (1971), Inelastic constitutive relations for solids - an internal-variable theory and its application to metal plasticity, Journal of the Mechanics and Physics of Solids, 19(6), 433455, doi:10.1016/0022-5096(71)90010-X.

Rice, J. R. (1975), Continuum mechanics and thermodynamics of plasticity in relation to microscale deformation mechanisms, in Constitutive Equations in Plasticity, edited by A. Argon, chap. 2, MIT Press, Cambridge, Mass.

Rice, J. R. (2002), Mechanics for a New Mellennium: Proceedings of the 20th International Congress of Theoretical and Applied Mechanics Chicago, Illinois, USA 27 August - 2 September 2000, chap. New Perspectives on Crack and Fault Dynamics, pp. 1-24, Springer Netherlands, Dordrecht.

Rice, J. R., C. G. Sammis, and R. Parsons (2005), Off-fault secondary failure induced by a dynamic slip pulse, Bulletin of the Seismological Society of America, 95(1), 109-134, doi: 10.1785/0120030166.

Rubin, A. M., and J.-P. Ampuero (2007), Aftershock asymmetry on a bimaterial interface, $J$. Geophys. Res., 112(B5).

Savage, H. M., and E. E. Brodsky (2011), Collateral damage: Evolution with displacement of fracture distribution and secondary fault strands in fault damage zones.

Scholz (2002), The mechanics of earthquakes and faulting, Cambridge University Press.

Scholz, C. H. (1998), Earthquakes and friction laws., Nature, 391, 37-42.

Semmane, F., F. Cotton, and M. Campillo (2005), The 2000 tottori earthquake: A shallow earthquake with no surface rupture and slip properties controlled by depth, J. Geophys. Res., 110(B3).

Shi, Z., and Y. Ben-Zion (2006), Dynamic rupture on a bimaterial interface governed by slipweakening friction, Geophysical Journal International, 165(2), 469-484.

Shipton, Z., and P. Cowie (2001), Damage zone and slip-surface evolution over $\mu \mathrm{m}$ to $\mathrm{km}$ scales in high-porosity navajo sandstone, utah, Journal of Structural Geology, 23(12), $1825-1844$.

Sibson, R. H. (1977), Fault rocks and fault mechanisms, Journal of the Geological Society of London, 133(3), 191-213.

Suzuki, T. (2012), Understanding of dynamic earthquake slip behavior using damage as a tensor variable: Microcrack distribution, orientation, and mode and secondary faulting, $J$. Geophys. Res., 117(B5).

Templeton, E. L., and J. R. Rice (2008), Off-fault plasticity and earthquake rupture dynamics: 1. dry materials or neglect of fluid pressure changes, J. Geophys. Res., 113(B9).

Thomas, M. Y., J.-P. Avouac, J.-P. Gratier, and J.-C. Lee (2014a), Lithological control on the deformation mechanism and the mode of fault slip on the longitudinal valley fault, taiwan, Tectonophysics, 632, 48-63.

Thomas, M. Y., J.-P. Avouac, J. Champenois, J.-C. Lee, and L.-C. Kuo (2014b), Spatiotemporal evolution of seismic and aseismic slip on the longitudinal valley fault, taiwan, Journal 
of Geophysical Research-solid Earth, 119, 5114-5139.

Vallage, A., Y. Klinger, R. Grandin, H. S. Bhat, and M. Pierrot-Deseilligny (2015), Inelastic surface deformation during the $2013 \mathrm{mw} 7.7$ balochistan, pakistan, earthquake, Geology, 43(12), 1079-1082.

Vermilye, J. M., and C. H. Scholz (1998), The process zone: A microstructural view of fault growth, Journal of Geophysical Research-solid Earth, 103(B6), 12,223-12,237.

Wald, D. J., and T. H. Heaton (1994), Spatial and temporal distribution of slip for the 1992 landers, california, earthquake, Bulletin of the Seismological Society of America, 84(3), 668691.

Walsh, J. B. (1965a), The effect of cracks in rocks on poisson's ratio, J. Geophys. Res., 70(20), 5249-5257.

Walsh, J. B. (1965b), The effect of cracks on the compressibility of rock, J. Geophys. Res., 70(2), 381-389.

Wang, L., S. Hainzl, M. Sinan zeren, and Y. Ben-Zion (2010), Postseismic deformation induced by brittle rock damage of aftershocks, Journal of Geophysical Research, 115(B10), B10,422.

Wang, Q., F. Feng, M. Ni, and X. Gou (2011), Measurement of mode i and mode ii rock dynamic fracture toughness with cracked straight through flattened brazilian disc impacted by split hopkinson pressure bar, Engineering Fracture Mechanics, 78(12), 2455-2469.

Wibberley, C. A., G. Yielding, and G. Di Toro (2008), Recent advances in the understanding of fault zone internal structure: A review, Geological Society, London, Special Publications, 299(1), 5-33.

Wilson, J. E., J. S. Chester, and F. M. Chester (2003), Microfracture analysis of fault growth and wear processes, punchbowl fault, san andreas system, california, Journal of Structural Geology, 25(11), 1855-1873.

Xu, S., Y. Ben-Zion, and J.-P. Ampuero (2012), Properties of inelastic yielding zones generated by in-plane dynamic rupturesii. detailed parameter-space study, Geophysical Journal International, 191(3), 1343-1360, doi:10.1111/j.1365-246X.2012.05685.x.

$\mathrm{Xu}$, S., Y. Ben-Zion, J.-P. Ampuero, and V. Lyakhovsky (2014), Dynamic ruptures on a frictional interface with off-fault brittle damage: Feedback mechanisms and effects on slip and near-fault motion, Pure and Applied Geophysics, 172(5), 1243-1267, doi:10.1007/s00024014-0923-7.

Yamashita, T. (2000), Generation of microcracks by dynamic shear rupture and its effects on rupture growth and elastic wave radiation, Geophysical Journal International, 143(2), 395-406.

Zhang, Q., and J. Zhao (2013), Effect of loading rate on fracture toughness and failure micromechanisms in marble, Engineering Fracture Mechanics, 102, 288-309.

Zhou, X., and A. Aydin (2010), Mechanics of pressure solution seam growth and evolution, Journal of Geophysical Research, 115(B12), B12,207. 